\title{
La reconstrucción de la teoría del razonamiento de Dignaga por medio de la lógica formal. Una crítica a la propuesta formalista del razonamiento*
}

\author{
HUANG XIANG \\ Departamento de Filosofía \\ Universidad Autónoma del Estado de Morelos \\ xiang@prodigy.net.mx
}

\begin{abstract}
Resumen: La propuesta formalista del razonamiento considera que los problemas filosóficos del razonamiento residen únicamente en los análisis formales de lógica. En este artículo, mediante el estudio de un caso histórico de la teoría del razonamiento de Dignaga, trato de demostrar que la propuesta formalista del razonamiento no es plausible, porque el problema de la corrección o incorrección de un razonamiento no sólo depende de las reglas lógicas, sino que también depende de los contextos prácticos en que se realiza este razonamiento. La teoría del razonamiento de Dignaga es una teoría que intenta estudiar la aplicación correcta de un razonamiento en sus contextos prácticos. Si reconstruimos este tipo de razonamiento por medio de una reducción a una lógica formal, lo hacemos al precio de sacrificar consideraciones sobre los contextos prácticos en que se realiza este tipo de razonamiento. La omisión de considerar estos contextos prácticos produce distorsiones o consecuencias paradójicas de nuestro entendimiento de la naturaleza de este tipo de razonamiento, tal como ocurre en las diferentes maniobras de reconstrucción de la teoría del razonamiento de Dignaga mediante su reducción a un tipo de lógica formal occidental.
\end{abstract}

Palabras clave: lógica de la antigua India, hetu, dirección inferencial, inducción

El razonamiento humano puede definirse como un conjunto de procesos cognitivos por medio de los cuales una persona infiere, a partir de un conjunto de información original que toma como premisas, otro conjunto de información que considera la conclusión. Así que un razonamiento se compone de tres partes: el proceso de inferencia, la información original y la información inferida. En el mundo occidental se considera tradicionalmente que los principios normativos que rigen el procedimiento inferencial mediante el que se obtiene válidamente una conclusión a partir de ciertas premisas es el objeto de estudio de la lógica. Como es bien sabido, la lógica estudia exclusivamente aspectos formales de las reglas normativas de las

${ }^{*}$ La primera versión de este trabajo fue un capítulo de mi tesis de doctorado. Agradezco tanto a Sergio Martínez Muñoz, director de la tesis, como a Carlos López Beltrán y Edna María Suárez Díaz, miembros del comité tutorial, que durante mi investigación sobre Dignaga siempre me alentaron y me ofrecieron sus valiosos comentarios y críticas. 
inferencias, sin ocuparse del contenido de las premisas ni de las conclusiones, de manera que la validez de una inferencia depende exclusivamente del uso correcto de las formas lógicas. Es decir, independientemente de que una premisa sea verdadera o falsa, se puede inferir legítimamente una conclusión si la estructura de la inferencia es formalmente válida. Llamamos a este concepto para modelar el razonamiento, que reduce los problemas del razonamiento a los análisis formales de lógica, la propuesta formalista del razonamiento.

La propuesta formalista tiene tres características importantes. La primera reside en que, si consideramos que los razonamientos humanos se conforman a las reglas abstractas de la lógica, los razonamientos humanos también son abstractos, es decir, no tienen nada que ver con los elementos concretos de sus contenidos. La segunda característica es que, dado que la validez de una regla lógica se establece independientemente de las influencias procedentes de los contenidos, los razonamientos humanos son esencialmente de carácter no contextual. El tercer rasgo viene del hecho de que los razonamientos humanos son normativos en el sentido de que prescriben la forma en que un sujeto racional puede pensar o actuar. De modo que, junto con los dos rasgos anteriores, la fuente de la normatividad del razonamiento también se ubica únicamente en las reglas lógicas que prescriben las relaciones formales entre las proposiciones y las que permiten realizar inferencias formales independientemente de su contenido.

A lo largo de la historia de la filosofía occidental, si bien no han faltado investigaciones sobre los razonamientos que no son reducibles a la propuesta formalista, tales como la abducción, la analogía, etc., la dirección formalista, junto con el desarrollo de la lógica, ha predominado en los estudios sobre el razonamiento humano. Por lo menos, según la interpretación tradicional que se ha hecho de Aristóteles, él intentó analizar el pensamiento y el discurso lógico especificando reglas de inferencia llamadas silogismos. Aristóteles sostenía que, en principio, los argumentos lógicos podían ser estudiados mediante la aplicación de una serie de silogismos cuya validez dependía únicamente de sus formas; por ello trató de encontrar todos los tipos de silogismos involucrados en el razonamiento lógico. La lógica estoica estudiaba el razonamiento humano enfocándose en las relaciones conectivas de las proposiciones, que eran independientes de lo que estas proposiciones decían. En los siglos posteriores a la época de Aristóteles y de los estoicos, varios filósofos trataron de profundizar este tipo de teoría del razonamiento; entre ellos destacaron especialmente Gottfried Leibniz, en el siglo XVII, con su teoría algebraica de los conceptos, y George Boole, en el siglo XIX, con su proyecto algebraico para representar las leyes del pensamiento. A principios del siglo xx, el gran desarrollo de la lógica encabezado por Frege y Russell reforzó este aspecto formalista del razonamiento; posteriormente han surgido numerosos sistemas formales 
que intentan caracterizar las inferencias deductivas como modelos de la racionalidad humana.

Por supuesto, los razonamientos humanos no se limitan únicamente a las inferencias deductivas. Tanto las investigaciones científicas como los razonamientos de la vida cotidiana utilizan las inferencias inductivas de manera imprescindible. Además, los razonamientos prácticos no son analizables únicamente mediante los razonamientos deductivos, sino que recurren a la teoría de la probabilidad para explicar las decisiones humanas. Los razonamientos inductivos son más problemáticos que los deductivos, no sólo por la dificultad de justificarlos de alguna manera, sino también por la variedad de interpretaciones ontológicas diferentes acerca de ellos. Sin embargo, se ha aceptado la idea de que los razonamientos inductivos, independientemente de las diferentes interpretaciones teóricas, deben adecuarse a los axiomas del cálculo de la probabilidad, formalizado por A.N. Kolmogorov, ya que violarlos nos lleva a la trampa desesperanzada de los libros de Holanda. Aunque las inferencias de la probabilidad no pueden reducirse a la lógica, los filósofos que sostienen la propuesta formalista insisten en la búsqueda de un paralelo entre las reglas probabilísticas y las reglas lógicas; esto es, se presupone que es posible encontrar una interpretación ontológica unívoca del cálculo probabilístico caracterizado por Kolmogorov que nos permitiría considerar la aplicación de las reglas de la probabilidad de acuerdo con el ideal formalista.

Aquí, todos los proyectos formalistas del razonamiento coinciden en que la lógica y la teoría de la probabilidad pueden modelar y explicar de manera fundamental y suficiente todos los razonamientos humanos y todas las conductas racionales. El positivismo lógico adoptó completamente esta creencia e intentó modelar los razonamientos de las investigaciones científicas a través de modelos expresados únicamente por las técnicas lógicas y probabilísticas. Esta creencia se replanteó de una manera ligeramente diferente en los años cincuenta, con el nacimiento de las disciplinas de la inteligencia artificial y las ciencias cognitivas. Los proyectos de inteligencia artificial, sobre todo al principio, intentaron construir programas computacionales que pudieran "razonar" y "tomar decisiones" por medio de cálculos lógicos, matemáticos y probabilísticos, a fin de modelar la inteligencia humana. Estos programas convertirían las reglas de la lógica en lenguaje computacional, lo que permitiría modelar el razonamiento de la misma manera que una computadora resuelve los problemas matemáticos. Así, en una primera etapa, las ciencias cognitivas utilizaron los programas de computadora para modelar de manera detallada y precisa todos los aspectos importantes del pensamiento humano. Durante la primera mitad del siglo $\mathrm{xx}$, la propuesta formalista había sido aceptada por muchos psicólogos. Por ejemplo, McCulloch y Pitts (1943) interpretaron la neurona como un circuito lógico, y Piaget propuso que el ser humano alcanza su 
pleno desarrollo intelectual cuando adquiere las operaciones formales, que no son sino la capacidad de razonar invocando principios lógicos. ${ }^{1}$ Detrás de todos estos intentos se presupone una imagen acerca de cómo funciona racionalmente la mente humana: el proceso del razonamiento es un tipo de cálculo mental que se conforma a las reglas prescritas por la lógica y el cálculo de probabilidades.

Sin embargo, a partir de los años sesenta, la propuesta formalista del razonamiento ha sido seriamente criticada. En el campo de la filosofía de la ciencia, los filósofos e historiadores de la ciencia han mostrado que el concepto de la racionalidad científica basado en la propuesta formalista no logra explicar adecuadamente las prácticas científicas que se observan en la historia de la ciencia, porque al aplicarse este enfoque, la mayoría de científicos deberían ser calificados de irracionales. Thomas Kuhn, Steven Toulmin y Paul Feyerabend, entre otros, argumentan que no se puede modelar la justificación y la selección de una teoría científica apelando únicamente a los análisis lógicos de las reglas metodológicas, ya que, por un lado, no existen las observaciones neutrales que permitan a los científicos realizar una evaluación con objetividad, y por otro, tampoco existe un conjunto de reglas aplicables universalmente a todas las situaciones, en especial cuando se trata de diferentes tradiciones de investigación en competencia (Newton-Smith 1981; Brown 1988). Muchos estudios recientes de filosofía e historia de la ciencia han mostrado desde diferentes perspectivas que la aplicación correcta de las metodologías y los razonamientos científicos tiene mucho que ver con distintos factores contextuales que son extralógicos. Por ejemplo, los historiadores de la ciencia sostienen que la justificación de una regla metodológica es un proceso histórico que tiene que ver con los propósitos específicos que los científicos persiguen en diferentes épocas y situaciones (Shapin y Shaffer 1985; Porter 1986; Pickering 1984; Biagioli 1993, entre otros). Los sociólogos de la ciencia muestran

${ }^{1}$ El mismo Piaget no sostuvo que los razonamientos humanos consistieran únicamente en las inferencias abstractas y no contextuales (Piaget 1972), pero su teoría ha sido considerada como argumento empírico a favor de la tesis de la lógica mental, según la cual, las reglas de los razonamientos no son más que los cálculos proposicionales, como lo describen Evans y Over: "La idea de que las leyes de la lógica no son distintas de las leyes del pensamiento ha gozado de una larga tradición filosófica y se estendió en la psicología por medio de la teoría piagetiana del pensamiento operacional formal (Inhelder y Piaget 1958) y por el trabajo de Henle (1962) [...]. Estos autores no propusieron modelos computacionales del tipo que esperarían los científicos del conocimiento hoy día; sin embargo, defensores de la lógica mental igualmente contemporáneos han intentado ofrecer precisamente explicaciones mejor especificadas de cómo razona la gente (véanse, por ejemplo, Braine y O’Brien 1991; Rips 1983, 1994)" (Evans y Over 1996, pp. 10-11). Manktelow y Over (1990, cap. 5) tienen una detallada descripción sobre la escuela de la lógica mental. Sobre la pregunta acerca de cuáles son los sistemas formales entre los muchos existentes que se utilizan para describir y prescribir los razonamientos humanos según la idea tradicional de la racionalidad, Manktelow y Over (1990) ofrecen un resumen breve pero muy bueno, que estudia separadamente la inferencia deductiva (cap. 4, pp. 63-84) y la inductiva (cap. 7, pp. 123-141). 
que los factores sociales, tales como los intereses personales e institucionales, las condiciones económicas, etc., pueden afectar decisivamente el establecimiento y la aplicación de una regla metodológica (Barnes 1974, 1977; Bloor 1976/1991; Collins 1985; Fuller 1989; Latour 1987; Pickering 1992... ). Filósofos como Kitcher (1993), Giere (1988) y Hutchins (1995) argumentan que las condiciones cognitivas de los científicos son factores importantes que delimitan el contexto en que la aplicación de una regla metodológica se considera apropiada. Además, una serie de estudios sobre la naturaleza de la tecnología y el experimento científico manifiestan que los factores tecnológicos también desempeñan un papel importante en la formación de muchas normas de la racionalidad científica (Hacking 1983; Franklin 1986, 1990; Galison 1987, 1997; Bijker, Hughes y Pinch 1987; Gooding 1990; Gooding, Pinch y Schaffer 1989; Pickering 1995, entre otros).

En el campo de las ciencias cognitivas, una serie de experimentos psicológicos acerca del razonamiento humano muestra que existe una tendencia a que los seres humanos razonen desviándose de las reglas abstractas establecidas por la lógica y las teorías de la probabilidad (Evans, Newstead y Byrne 1993; Evans y Over 1996; Kahneman, Slovic y Tversky 1982; Manktelow y Over 1990, 1993; Stanovich 1999; Stein 1996...). A través de diferentes tipos de estudios acerca de estos experimentos, muchos psicólogos argumentan que la aparente discrepancia entre los razonamientos que los seres humanos utilizan en su vida cotidiana y las reglas normativas establecidas por la lógica y las teorías de la probabilidad implica que debemos abandonar el concepto tradicional de la racionalidad basada en la propuesta formalista. En vez de este concepto tradicional de la racionalidad, una alternativa plausible es la tesis contextualista del razonamiento según la cual, para poder entender adecuadamente la racionalidad, es importante modelar los diferentes tipos de razonamiento tomando en cuenta los diferentes factores que configuran un contexto particular de aplicación de un razonamiento específico. Estos factores contextuales, tales como la limitación biológica de la capacidad cognitiva, los propósitos prácticos para realizar un razonamiento, las condiciones tecnológicas y sociales, las situaciones discursivas, etc., contribuyen a la decisión de si es adecuada o no la aplicación de una regla normativa del razonamiento.

Mediante el estudio de un interesante caso de la historia de la lógica de la India antigua, mi trabajo intenta ofrecer un ejemplo que apoya, desde una perspectiva diferente, estas críticas a la propuesta formalista del razonamiento.

Durante la segunda parte del siglo $\mathrm{xx}$, sosteniendo la propuesta formalista del razonamiento, la mayoría de los historiadores de la lógica ha tratado de evaluar el logro de los antiguos lógicos, teniendo como criterio de evaluación la lógica moderna, como lo muestran las obras de W. Kneale 
y M. Kneale (1962), I.M. Bochenski (1956) y J. Lukasiewicz (1957). Lo que ellos nos señalan es cómo se esforzaron enormemente los antiguos lógicos para lograr formalizar el razonamiento, cosa que sólo los lógicos del siglo Xx han alcanzado. Lo mismo ocurre con los estudios históricos sobre el desarrollo de la lógica en la India antigua, los cuales tratan de reconstruir las antiguas teorías orientales comparándolas con el desarrollo de la lógica en Occidente, determinando, por ejemplo, en qué momento histórico una teoría de la lógica india alcanzó algún punto técnico al que la lógica occidental había llegado. Obviamente, la comparación entre los diferentes sistemas de la lógica puede, de una u otra manera, ilustrar ciertos aspectos interesantes y hasta sorprendentes de estos sistemas. Sin embargo, un problema para estos estudios históricos es que, al estar basados en la propuesta formalista, pasan por alto diferentes tipos de factores contextuales de una inferencia que, como las críticas a la propuesta formalista señalan, son muy importantes para un modelo adecuado del razonamiento. Así, durante estos procesos de reconstrucción, muchos caracteres importantes e interesantes de la lógica india han sido completamente olvidados o distorsionados. En este trabajo analizaré un ejemplo de este tipo de distorsión a través de un caso concreto de la teoría del razonamiento de Dignaga, quien, según Bochenski, "es el más grande lógico de la India, sin duda" (1956, p. 433). Quiero mostrar que la teoría del razonamiento de Dignaga intenta modelar un tipo de razonamiento discursivo que se aplica en una argumentación donde los rivales no comparten todas las premisas. Un rasgo distintivo de esta teoría es que, para Dignaga, la dirección inferencial de este tipo del razonamiento va de una conclusión a las razones que la apoyan, un rasgo que, como veremos, no puede capturarse por medio del enfoque formalista. ${ }^{2}$

En la primera sección, utilizando el ejemplo de la obra de Bochenski, analizaré cómo, con el propósito de encontrar una línea de desarrollo de la lógica formal en la India antigua, los historiadores occidentales reconstruyen la teoría del razonamiento de Dignaga reduciéndola al silogismo occidental. En la segunda sección trataré de exponer la teoría del razonamiento de Dignaga desde una perspectiva diferente que considero más cercana a su espíritu original. En la tercera sección argumentaré que, debido al hecho de que la teoría de Dignaga es una teoría del razonamiento inductivo que intenta modelar los procesos de razonamiento discursivo, cuya característica importante es que la dirección de la inferencia va de una conclusión a las razones que la apoyan, cualquier reconstrucción de

\footnotetext{
${ }^{2}$ Es posible que ciertas versiones de la lógica no monotónica sean más eficientes para interpretar la lógica de la India antigua, cosa que no voy a explorar aquí (Oetke 1996). Sin embargo, es importante ver que una interpretación adecuada no debe basarse en la propuesta formalista, ya que la idea no contextual pasa por alto factores contextuales que contribuyen al establecimiento de los principios normativos de la lógica de la India antigua.
} 
esta teoría que la reduzca al esquema formal estará condenada al fracaso. Si mi estudio de este caso es correcto, servirá como un apoyo a la tesis contextualista del razonamiento y, en consecuencia, será un contraejemplo para la propuesta formalista del razonamiento.

\section{La reconstrucción de la teoría del razonamiento de Dignaga de acuerdo con la lógica occidental}

El caso más famoso de reconstrucción de la teoría del razonamiento de Dignaga por medio de la lógica occidental es la obra monumental de Stcherbatsky (1930-1932), que hace una detallada comparación entre el silogismo aristotélico y la regla trimembre (trairupya) de Dignaga. Sin embargo, en este trabajo no voy a exponer su trabajo por las siguientes dos razones. La primera es que, como señala D.J. Kalupahana (1992, p. 281), los recursos del estudio de Stcherbatsky se basan en los comentarios de Dharmakirti, quien, aunque considerado discípulo de Dignaga, sostiene una epistemología y una metafísica muy diferentes de las que sostiene su maestro. La confusión entre las posturas de estos dos filósofos ha sido un obstáculo importante para entender la teoría del razonamiento de Dignaga. La segunda razón es que, como Stcherbatsky no estaba muy familiarizado con el desarrollo de la lógica moderna que había tenido lugar a principios del siglo Xx, su libro fue tachado por el lógico Bochenski de contener "tal cantidad de errores en cuestiones de Lógica sistemática, que sus resultados necesitan una revisión detallada y profunda" (Bochenski 1956, p. 435). Por eso considero que es mejor analizar aquí la reconstrucción de Bochenski, un trabajo que, a pesar de su reducido tamaño, resulta ser técnicamente mucho más sofisticado que el de Stcherbatsky.

De acuerdo con Bochenski, en la India antigua se desarrolló una lógica formal de clases y de predicados que corresponde más o menos al silogismo aristotélico. Este tipo de lógica formal, que tuvo su origen en la escuela de Nyaya (Naiyayika), fue desarrollada sistemáticamente, por primera vez, por el budista Dignaga (480-540 d.C.); culminó con la formulación de otro budista, Dharmakirti (600-680 d.C.); y, finalmente, fue heredada y discutida por otras escuelas filosóficas de la India, entre las cuales sobresalieron la escuela de Jaina y la de Navya-Nyaya, o nuevo Nyaya.

En el siglo VI a.C., debido al establecimiento de los estados independientes en el norte de la India, el monopolio ideológico del brahmanismo fue desafiado por el surgimiento de diferentes escuelas filosóficas y religiosas. Como consecuencia de los frecuentes contactos y discusiones entre estas escuelas, surgieron problemas acerca de la argumentación justa y efectiva. La escuela Nyaya consideraba la inferencia como uno de los instrumentos cognitivos para obtener conocimientos verdaderos; por lo tanto, empezó a investigar los problemas del razonamiento y la argumentación. Finalmen- 
te, en el siglo II d.C., apareció Nyaya-sutras de Aksapada Gautama, la obra monumental que "se convirtió en la base de todo el pensamiento lógico de la India" (Bochenski 1956, p. 432). ${ }^{3}$ En la parte I.1.32-I.1.39 estudia la fórmula de inferencia citada por Bochenski:

32. Los "miembros (avayavah) [de la inferencia] son: Tesis (pratijña), razón (hetu), confirmación (ejemplo, udaharana), aplicación (upanaya) y conclusión (nigamana).

33. La tesis es la declaración de lo que hay que demostrar.

34. La razón es la demostración de lo que hay que demostrar, mediante el carácter homogéneo o afirmativo de la confirmación.

35. Igualmente, mediante el carácter heterogéneo o negativo (de la confirmación).

36. Ejemplo (drstanta) homogéneo (o afirmativo) es una confirmación que se sabe que posee la propiedad (dharma) que hay que demostrar, y que implica que esta propiedad se encuentra invariablemente en la razón.

37. Ejemplo heterogéneo (o negativo) es una confirmación que se sabe que carece de la propiedad que hay que demostrar, y que implica que la ausencia de esta propiedad se rechaza invariablemente en la razón dada.

38. Aplicación es la determinación, respecto de la confirmación, de lo que se va a demostrar que "es así" o "no es así".

39. Conclusión es la repetición de la proposición una vez aducida la razón. ${ }^{4}$

De tal manera que una inferencia se puede expresar por medio del siguiente esquema de cinco pasos:

(N1) Tesis: En la montaña hay fuego;

(N2) Fundamentación: porque hay humo en la montaña;

(N3) Confirmación: como en una cocina, no como en un estanque;

(N4) Aplicación: es así;

${ }^{3}$ Aquí sólo repito lo que Bochenski considera correcto. En lugar de "Gautama", algunos textos lo traducen como "Gotama". Acerca de la fecha de la aparición de Nyaya-sutras, ha habido mucha controversia. Pocos historiadores creen que es una obra escrita por un autor llamado Aksapada Gautama. La mayoría considera que más bien se trata de una obra colectiva de los discípulos de la escuela Nyaya (H.P. Sastri 1905, 1906; S. Dasgupta 1922, pp. 276-280). Además, Aksapada y Gautama pueden ser dos personas; por ejemplo, M.S.Ch. Vidyabhusana cree que Gautama hizo la primera parte de Nyaya-sutras en 550 a.C, y Aksapada escribió la versión que ahora podemos fechar en 150 d.C. (1913, pp. i-ix).

${ }^{4}$ Bochenski 1956, p. 443. La versión traducida a idioma occidental que Bochenski adopta es la alemana, publicada en 1929 por W. Ruben. Las recientes versiones en idioma occidental son la de D. Chattopadhyaya y M. Gangopadhyaya (1967), donde se encuentra el texto citado y sus comentarios en las páginas 105-120. El problema más grave de la traducción de Bochenski es que utiliza "confirmación" para traducir "udaharana" — su otra opción sería "ejemplo", que es más aceptable-, porque existen otros estudios que utilizan la palabra "confirmación" para traducir "upanaya", que es la "aplicación" en el caso de Bochenski. Un ejemplo es la obra de N.S. Junankar (1978, p. 258). 
(N5) Conclusión: luego es así.

Bochenski interpreta este esquema considerando que es un razonamiento en que un agente ofrece razones para defender una tesis:

A: Afirmo que F conviene a M (N1).

B: ¿Por qué?

A: Porque $\mathrm{H}$ conviene a S (N2).

B: ¿Y qué?

A: Pues verá Ud.: a E le convienen $\mathrm{H}$ y $\mathrm{F}$ a la vez, a $\mathrm{E}$ no le convienen ni $\mathrm{H}$ ni $\mathrm{M}$ (N3). Ahora bien, así es también nuestro caso (N4). Luego F conviene a M (N5).

Nuestro "silogismo" tiene justamente esta misma forma. ${ }^{5}$

Estoy de acuerdo con esta interpretación del esquema de cinco pasos, pero dudo de la afirmación de Bochenski que sostiene que el silogismo occidental, una inferencia deductiva cuya conclusión se sigue necesariamente de las premisas, tiene la misma forma que el esquema mencionado, en el que son los ejemplos particulares los que justifican la conclusión. Según Bochenski, el razonamiento representado por el esquema anterior pertenece a un tipo llamado "deducción por analogía". Dado que en la filosofía india antigua es difícil encontrar la concepción de la universalidad, en lugar de la premisa mayor del silogismo occidental, la teoría de Nyaya simplemente pone un caso como ejemplo analógico. En la época de Nyaya-sutras no existía todavía en la India una lógica formal. ${ }^{6}$ Para que la teoría del razonamiento de Nyaya pueda convertirse en una lógica formal, además de sustituir la justificación por la analogía, habría la dificultad de eliminar los pasos (N4) y (N5), los cuales no pueden encontrarse en el silogismo aristotélico. ${ }^{7}$ De acuerdo con Bochenski, fue Dignaga quien hizo un desarrollo decisivo para superar estas dos dificultades.

${ }^{5}$ Bochenski 1956, p. 446. Aquí hago un cambio de los símbolos de Bochenski para relacionar mejor este esquema abstracto con el caso concreto del fuego en la montaña. Obviamente, estos símbolos no son variables a la manera como los entiende la lógica occidental, sino que son los casos particulares. Se puede parafrasear el esquema expresado en un texto de K.H. Potter: "Usted y yo estamos en una ladera que domina un valle, y vemos que de otra ladera que está frente a nosotros sube una nube de humo. 'Hay fuego en esa colina', le digo, 'porque hay humo. Usted sabe, porque ha visto antes que las cosas con fuego humean; por ejemplo, las cocinas, y, además, nunca se ve humo donde no hay fuego, como en un lago.'"] (Potter 1963, p. 57).

${ }^{6}$ Un caso brillante de análisis detallado de la teoría del razonamiento de Nyaya sin ningún intento de compararlo y reconstruirlo a través de la lógica occidental, aunque tuvo que usar las nomenclaturas de la filosofía occidental, es la obra de N.S. Juanankar (1978), uno de los mejores estudios occidentales sobre la filosofía de Nyaya.

${ }^{7}$ Para ver con más claridad el problema de los pasos (N4) y (N5), veamos el siguiente comentario de Ch. Sharma, quien cree que, o bien estos dos pasos o bien los dos primeros (N1) 
La plausibilidad de reducir los cinco pasos del esquema del razonamiento de Nyaya a un esquema del razonamiento de tres pasos fue planteada por los budistas que estudian la "inteligencia de la razón" (hetuvidya, donde hetu = razón; vidya = inteligencia o conocimiento). Mientras que la escuela Nyaya estudiaba los problemas del razonamiento dentro del brahmanismo, el pensamiento ortodoxo en la India de aquella época, la escuela Hetuvidya estudiaba los mismos problemas dentro de la escuela budista. Los primeros filósofos budistas aceptaban el esquema de razonamiento de Nyaya; sin embargo, en las obras de la época Mahayana empezaron a aparecer los tratados que intentan usar una inferencia trimembre, i.e., un esquema del razonamiento de tres pasos, para representar un razonamiento. ${ }^{8}$ Bochenski menciona a Vasubandhu (siglo IV d.C.); también se observa este intento en las obras de Maitreya y Asanga de la misma época. Sin embargo, fue Dignaga quien hizo una defensa a favor de la inferencia trimembre. Bochenski trata de reconstruir de la siguiente manera lo que Dignaga quiere decir:

El silogismo trimembre de Dignaga consta, por consiguiente, de fundamentación, ejemplos y tesis, e.d., de segundo, tercero y primer miembros del silogismo pentamembre clásico, y precisamente por este mismo orden; quedan excluidas la aplicación (N4) y la conclusión (N5). Por lo tanto, después de la reducción, el ejemplo clásico quedaría así:

y (N2), pero no ambos, son esencialmente redundantes: "Si lo comparamos [el esquema] con el silogismo aristotélico, que sólo tiene tres proposiciones, encontraremos que este silogismo de Nyaya corresponde al modo Barbara (AAA) de la primera figura, que es el modo más fuerte de la figura más fuerte. Aunque el silogismo de Nyaya tiene cinco proposiciones y el aristotélico sólo tres, los términos en ambos son sólo tres —el sadhya o mayor, el paksa o menor, y el hetu o medio. De las cinco proposiciones, dos parecen redundantes y fácilmente podemos omitir las dos primeras o las dos últimas, las cuales son esencialmente iguales. La primera coincide con la quinta y la segunda con la cuarta. Si omitimos las dos últimas, las tres restantes corresponderán a la conclusión, la premisa menor y la premisa mayor, en ese orden. O bien, si omitimos las dos primeras, las otras tres corresponderán a la premisa mayor, la premisa menor y la conclusión del silogismo aristotélico. De ahí que si omitimos los dos primeros miembros del silogismo de Nyaya según esta segunda opción, encontraremos que se asemeja al silogismo aristotélico de la primera figura: (1) Todo lo que tiene humo tiene fuego (premisa mayor); (2) Esta montaña tiene humo (premisa menor); (3) Por lo tanto, esta montaña tiene fuego (conclusión). Y el típico silogismo aristotélico se puede plantear en la forma de Nyaya así: (1) Sócrates es mortal (pratijña); (2) porque es un hombre (hetu); (3) todo el que es hombre es mortal, por ejemplo, Pitágoras (udaharana); (4) Sócrates es un hombre que es invariablemente mortal (upanaya); (5) por lo tanto, Sócrates es mortal (nigamana)" (Sharma 1960, p. 198-199).

${ }^{8}$ Generalmente se considera que después de que Buda creó el budismo en el siglo v a.C., y antes de que desapareciera de la India en el siglo x d.C., esta escuela religiosa había pasado por tres importantes etapas, clasificadas de acuerdo con el cambio de sus principios básicos: la etapa de Hinayana, siglo v a.C.-siglo I d.C; la primera etapa de Mahayana, siglo I-siglo v d.C., y, finalmente, la segunda etapa de Mahayana, siglo v-siglo x d.C. Una breve introducción de los cambios filosóficos puede verse en Stcherbatsky 1930-1932, vol. I, pp. 1-14. 
Fundamentación: En la montaña hay humo;

Confirmación: Como en la cocina en la cual hay fuego, no como en el estanque, en el que no lo hay.

Tesis: Luego, en la montaña hay fuego. (1956, p. 451)

Lo que queda claro es que Dignaga, al eliminar los pasos (N4) y (N5) del esquema del razonamiento de Nyaya, lo convierte en un esquema del razonamiento de tres pasos. Sin embargo, la traducción del texto y la exposición de Bochenski sugiere que Dignaga estaba intentando encontrar un tipo de inferencia equivalente al silogismo aristotélico, algo que no es correcto.

En primer lugar, veamos la traducción. En su versión del texto de Dignaga, Bochenski utiliza varias veces terminología de la lógica occidental, como "deducción", "silogismo", "deducción válida" y "deducción indudablemente válida", lo que sugiere que Dignaga estaba hablando de las ideas a las que se refieren estas expresiones. Sin embargo, en el texto citado, lo que Dignaga analiza es una inferencia (anumana), por la cual investigamos, obtenemos y defendemos conocimientos verdaderos. Para la mayoría de los filósofos de la India antigua — sobre todo para Dignaga, como veremos en la siguiente sección-, estas inferencias siempre requieren un proceso inductivo para que la razón (hetu) se establezca. Muchos historiadores y filósofos occidentales utilizan "inferencia" para traducir la palabra "anumana", como lo hacen Th. Stcherbatsky (1930-1032), N.S. Junankar (1978), N. Smart (1964) y Ch. Sharma (1960), entre otros, pero cuando ellos utilizan "silogismo" para referirse al esquema del razonamiento de tres pasos que Dignaga usa en este texto, lo hacen pensando simplemente en su conveniencia para los lectores occidentales, y no quieren decir que una inferencia tripitaka es lo mismo que el silogismo de la lógica occidental. ${ }^{9}$ Una gran diferencia entre una tripitaka y un silogismo es que tienen diferentes maneras de entender la validez. En la lógica occidental, un silogismo es válido si y sólo si la conclusión se sigue necesariamente de sus premisas. En las diferentes escuelas de filosofía india, donde se considera la inferencia (anumana) como un recurso para conseguir y defender conocimientos verdaderos, la validez de una inferencia depende de su uso

${ }^{9}$ Como dice Ch. Sharma de manera muy clara: "Esto es inferencia. La lógica india no separa la deducción de la inducción; la inferencia es un proceso complejo que incluye ambas. La lógica india también rechaza la perspectiva verbalista de la lógica; estudia los pensamientos como tales y no las formas de pensamiento solas. La lógica formal y la material están mezcladas aquí" (1960, p. 198). R.S.Y. Chi explica por qué usa "silogismo" en vez de otras alternativas para referirse a las inferencias de la lógica de la India antigua: "Ni el silogismo pentamembre ni el trimembre pueden coincidir con precisión con la forma conocida del silogismo que tiene una premisa menor, una premisa mayor y una conclusión. Es posible acuñar un nuevo conjunto de símbolos y fórmulas para la lógica india si nos apegamos a la estructura sintáctica del silogismo indio; pero sería extremadamente inconveniente, engorroso e inútil." (Chi 1969, p. 15) 
correcto. Por lo tanto, para los filósofos de la India antigua, una inferencia es "abadhita", la palabra sánscrita que Bochenski traduce por "válida", cuando no comete los errores que transgreden los principios normativos del uso correcto de un razonamiento. Por lo tanto, cuando Bochenski usa la locución "deducción indudablemente válida", debemos entender como "una inferencia que no transgrede los principios normativos del uso correcto de un razonamiento". Además, como veremos en la siguiente sección, esta inferencia es de carácter más inductivo que deductivo.

Ahora veamos los errores que Bochenski comete en la exposición de las teorías de Dignaga. Para Bochenski, Dignaga no sólo elimina del esquema del razonamiento los pasos (N4) y (N5), sino que también cambia el orden de los primeros tres pasos. Como hemos visto anteriormente, en el esquema pentamembre de Nyaya, (N1) es la tesis que requiere defenderse, (N2) la razón y (N3) la confirmación en la terminología de Bochenski. No obstante, en la reconstrucción del esquema trimembre de Dignaga, Bochenski reorganiza los tres pasos en el siguiente orden: la razón, la confirmación y la tesis, que corresponden a la premisa menor, la premisa mayor y la conclusión de un silogismo aristotélico, respectivamente. Una vez que la confirmación se convierte implícitamente en una proposición universal, podemos tener una inferencia que se identifica con el silogismo aristotélico. Sin embargo, en todas las presentaciones originales de las obras de Dignaga, el orden de los tres pasos del razonamiento siempre es así: primero la tesis, posteriormente, se ubica la razón y en tercer lugar la confirmación (o mejor dicho, el ejemplo). Este orden representa el procedimiento de inferencia cuya dirección va de la tesis, que es la conclusión de acuerdo con la terminología de la lógica occidental, a las razones, que son las premisas. Como veremos en la tercera sección, las diferentes direcciones de la inferencia muchas veces implican diferentes procesos psicológicos y epistémicos de un razonamiento. Eso es lo que ocurre en nuestro caso. Aparentemente, la reorganización del orden de la inferencia del esquema trimembre de Dignaga está de acuerdo con el silogismo aristotélico, cuya dirección inferencial va de premisas a conclusión, y para Bochenski esta maniobra podría ser conveniente para exponer el proceso del nacimiento de la lógica formal en la India. Sin embargo, como veremos en las siguientes dos secciones, según Dignaga, el orden correcto del razonamiento es un requisito importante para realizar una inferencia trimembre. Si lo pasamos por alto, se dejan de lado los compromisos prácticos que son elementos constitutivos de un razonamiento.

Una vez que evitamos los posibles malentendidos causados por las traducciones y exposiciones problemáticas de Bochenski, podemos estar de acuerdo con él cuando dice que fue Dignaga quien trató de defender la tesis de que el esquema de tres pasos es suficiente para modelar una inferencia apropiada y válida tal y como la entendía la filosofía india antigua. ¿Qué 
razones da Dignaga para sostener su tesis? Para Bochenski, estas razones se resumen en la regla trimembre (trairupya) y la rueda de las razones (hetucakra), los dos conceptos cruciales requeridos para entender la teoría de Dignaga.

Según Bochenski, la regla trimembre comprende las siguientes tres reglas:

(1) $H$ está presente en $M$ (el humo en la montaña);

(2) $H$ está presente en los ejemplos positivos, en nuestro caso, la cocina (el humo en la cocina que tiene fuego);

(3) $H$ no está presente en los ejemplos negativos, en nuestro caso, el estanque (no hay humo en el estanque, el cual no tiene fuego);

Luego, $F$ está presente en $M$. (1956, p. 451, con algunos cambios de símbolo.)

Bochenski resume de la siguiente manera la rueda de las razones:

Los "dos modos posibles" [...] en los sapaksas [ejemplos positivos] son: (1) que $H$ sea inherente a todos, (2) que $M$ sea inherente a algunos; a los que hay que añadir todavía la tercera posibilidad no citada aquí, a saber, (3) que la marca no sea inherente a ningún sapaksa. Por ellos, habla Dignaga de "tres casos posibles". Respecto de los vipaksas [ejemplos negativos], hay igualmente tres casos, en cuanto que la marca puede ser inherente a todos ellos, a algunos o a ninguno. Con lo cual resultan nueve casos posibles. Representando estos tres casos con "A", "I" y "E" se obtiene el siguiente cuadro en el que la primera letra se refiere a los sapaksas y la segunda a los vipaksas:

$\begin{array}{rrrrrr}\text { 1. } & \text { A A } & \text { 2. } & \text { A E } & \text { 3. } & \text { A I } \\ \text { 4. } & \text { E A } & \text { 5. } & \text { E E } & \text { 6. } & \text { E I } \\ \text { 7. } & \text { I A } & \text { 8. } & \text { I E } & \text { 9. } & \text { I I }\end{array}$

De estos modos, solamente dos, el 2 y el 8, son válidos (1956, p. 452).

La exposición de Bochenski de estos dos conjuntos de reglas, el de la regla trimembre y el de la rueda de las razones, resulta ser demasiado breve; tampoco profundiza el autor en las razones de por qué estos dos conjuntos de reglas ayudan a Dignaga a sostener que el esquema del razonamiento de tres pasos es suficiente para una inferencia válida y apropiada. Éstas son cuestiones de las que me ocuparé en la próxima sección. Sin embargo, se puede observar que, según Bochenski, lo que Dignaga trata de encontrar mediante la regla trimembre y la rueda de la razón son las reglas generales de las regularidades entre el término medio y el término mayor, de manera que sugiere que, con estos recursos, Dignaga había logrado dar un paso decisivo hacia la formulación de una premisa mayor en el sentido del silogismo aristotélico. No obstante, desde esta perspectiva, el punto defectuoso de la teoría de la inferencia de Dignaga es que se encuentra todavía 
totalmente determinada por los ejemplos (Bochenski 1956, p. 454). De acuerdo con la interpretación de Bochenski, este problema fue superado por un discípulo de Dignaga, Dharmakirti, quien introdujo "en el trairupya la palabra 'eva' ('sólo'), con lo que la confirmación se convierte en una cosa completamente distinta de lo que había sido hasta este momento: de mera aducción de ejemplos se convierte en una premisa universal" (1956, p. 448).

De acuerdo con Dharmakirti, el esquema de inferencia se debe formular así:

$\left(N^{\prime} 1\right)$ Tesis: En la montaña hay fuego;

(N'2) Fundamentación: porque hay humo en la montaña;

$\left(\mathrm{N}^{\prime} 3\right)$ Confirmación: cuando se encuentra humo se encuentra fuego, como en una cocina; cuando no se encuentra fuego no se encuentra humo, como en un estanque.

Se ve muy claro que la confirmación ya es una premisa universal acompañada por dos ejemplos concretos. Tanto Dharmakirti como sus discípulos trataban de sostener la tesis de que en el esquema mencionado arriba, los ejemplos deben ser prescindibles. De manera que, finalmente, logró una forma definitiva idéntica al silogismo. Dice Bochenski:

Su formulación [...] recuerda más bien una fórmula de la Lógica matemática moderna que el silogismo ockhamiano:

Para todo $x$ : si $x$ es $A$, entonces $x$ es $B$;

Ahora bien, $a$ es $A$;

Luego $a$ es $B$. (1956, p. 456)

Según Bochenski, de esta forma culminó el desarrollo de la lógica india antigua hacia una lógica formal. ${ }^{10}$

Lo que a continuación quiero demostrar es que esta reconstrucción de la teoría del razonamiento de Dignaga la distorsiona gravemente. La teoría del razonamiento de Dignaga, al contrario de lo que dice Bochenski, es una

${ }^{10}$ Aunque en esta sección utilizo únicamente la obra de Bochenski, muchos historiadores contemporáneos de la filosofía india antigua sostienen una idea similar. Por ejemplo, en una introducción reciente de la filosofía budista india, S.R. Bhatt dice: "La contribución más innovadora de Dignaga es que presenta un esquema formal de diferentes relaciones del término medio con el término mayor (hetucakra) y resalta las condiciones de validez de la inferencia en ese terreno. Con esto intenta construir un sistema formal de lógica. La teoría de la inferencia de Dignaga fue expuesta detalladamente y desarrollada más a fondo por Dharmakirti." (1997, p. 427) 
teoría del razonamiento diferente y consistente, cuyo esquema de inferencia no puede ser reconstruido por medio del silogismo. Para demostrarlo, expondré con detalle la teoría de Dignaga en la siguiente sección.

\section{La teoría del razonamiento de Dignaga}

La idea filosófica central de Dignaga está contenida en Pramana-samucca$y a$, donde el autor estudia los problemas epistémicos y metafísicos de la escuela yogacara (o vijñaptimatrata). Esta obra monumental ha sido preservada en sánscrito y tibetano, y fue introducida al mundo occidental y estudiada críticamente en el decenio de 1930 por Stcherbatsky (19301932), Randle (1930) y Mookerjee (1935), entre otros. Si bien en esta obra las principales tesis epistémicas y metafísicas de Dignaga han sido expresadas detalladamente, como se puede ver en las obras de los autores occidentales mencionados antes, la parte sobre la teoría del razonamiento es bastante breve y hasta confusa, como se manifiesta en los textos citados por Bochenski. Los estudios occidentales sobre la teoría del razonamiento de Dignaga se basan generalmente en el comentario de su discípulo Dharmakirti, llamado Nyaya-bindu, lo cual ha distorsionado de manera crucial la teoría del razonamiento de Dignaga. ${ }^{11} \mathrm{El}$ malentendido de los estudiosos occidentales sobre la teoría de la inferencia de Dignaga no causa extrañeza si consideramos el hecho de que los dos tratados de Dignaga sobre la teoría de la inferencia, Nyaya-pravesa y Nyayamukha, donde se analiza la regla trimembre (trairupya) y la rueda de las razones (hetu-cakra), no han sido preservados en sánscrito, sino que se conocen sólo a través de las traducciones tibetanas y chinas. ${ }^{12}$

${ }^{11}$ De hecho, Dharmakirti es el discípulo de un discípulo de Dignaga, y no tuvo la oportunidad de aprender personalmente con Dignaga. Sobre la relación personal entre Dharmakirti y Dignaga, véase Stcherbatsky 1930-1932, vol. I, pp. 31-37. Sin embargo, Stcherbatsky, como muchos otros historiadores occidentales, considera equivocadamente que las obras de Dharmakirti son fieles interpretaciones de la teoría del razonamiento de Dignaga.

${ }^{12}$ En China, Xuan Zang (600-664), filósofo budista contemporáneo de Dharmakirti, tradujo al chino Nyayamukha en el año 647 y Nyaya-pravesa en 649; además, impartió cátedra acerca de la teoría de la inferencia basada en los dos tratados de Dignaga. Las notas y los comentarios de sus discípulos de estas clases resultaron ser bastante fructíferos. Entre ellos sobresale el comentario monumental sobre Nyaya-pravesa, llamado "El gran comentario sobre Nyaya-pravessa" (Yinming Ruzhengli Dashu), hecho por Kui Ji (632-682). Otros importantes comentarios son "El comentario de Nyaya-pravessa" (Yinming Ruzhengli Lunshu) de Wen Gui, "La cítica sobre Nyaya-pravessa" (Yinming Ruzhengli Yiduan) de Hui Zhao, "Las notas sobre Nyaya-pravessa" (Yingming Ruzhengli Qianhouji) de Zhi Zhou y "El comentario sobre Nyayamukha" (Yinming Zhenglimenlun Shuji) de Shen Tai. Mi exposición de la teoría de la inferencia de Dignaga en lo que sigue se basa principalmente en estas fuentes. Para una breve introducción a estas fuentes chinas, véase Chi 1969, pp. lxix-lxxviii y 189). En 1927 y 1930 se publicó la obra Nyaya-pravesa (Gaekwad Oriental Series, Baroda), que, conforme a la tradición del budismo tibetano, es una obra de Dignaga (Dhruva 1930; Bhattacharya 1927). Sin embargo, según la tradición del budismo chino, es una obra de Sankarasvamin (siglo vi d.C.), otro 


\subsection{La función de la teoría del razonamiento de Dignaga}

Para Dignaga, sólo existen dos orígenes del conocimiento: el directo es por medio de la experiencia (pratyaksa) y el indirecto es por medio del razonamiento (anumana). En Pramana-Samuccaya, Dignaga se dedica a defender esta tesis con su complicada metafísica y teoría de la epistemología. En los tratados Nyayamukha y Nyaya-pravesa, Dignaga expone con detalle su teoría del razonamiento. Existen dos formas en las que una persona puede razonar para adquirir conocimiento: por un lado, un agente puede llevar a cabo un razonamiento para sí mismo (svabhavanumana); por otro, un razonamiento puede ser utilizado por el agente en argumentaciones contra otros, ya sean compañeros de la misma escuela filosófica o rivales de las otras escuelas (pararthanumana). Un razonamiento del primer tipo puede realizarse mediante los procesos mentales del agente; pero para el segundo tipo, el agente tiene que expresar su proceso mental oral o literariamente (Barlingay 1965, p. 107; Stcherbatsky, 1903-1932, vol. I, p. 275). ${ }^{13}$ Dado que estas dos formas de razonamiento tienen diferentes compromisos contextuales, existen diferentes reglas para su realización en la práctica. Sin embargo, ambas formas tienen que seguir la regla trimembre y la rueda de la razón. Mientras que en Pramana-Samuccaya el autor destaca la primera forma, en sus tratados sobre el razonamiento se dedica a la segunda. En lo que sigue también me dedico a la segunda forma.

La cuidadosa distinción entre estos tipos de razonamiento significa que para Dignaga es importante que una teoría del razonamiento incorpore los factores contextuales y prácticos de la aplicación de un razonamiento. Eso también se ve en su caracterización de las funciones de las razones en los contextos discursivos de un razonamiento. Según Dignaga, hay que ver el problema desde dos perspectivas diferentes. Desde la perspectiva de un agente $p_{1}$ que defiende su tesis durante un proceso de argumentación, la razón (hetu) funciona para ayudar a que su rival $p_{2}$ entienda por qué la tesis está justificada o bien apoyada. A esta función de iluminar el entendimiento por parte del agente $p_{1}$ que defiende su tesis, se la llama razón iluminativa (upatti-hetu). Desde la perspectiva del oponente $p_{2}$ en el mismo procedimiento de argumentación, la razón ayuda para comprender por qué la tesis en cuestión está justificada o bien apoyada. A esta función del entendimiento por parte del oponente se la llama razón comprensiva

lógico budista que, si bien no estaba de acuerdo completamente con la teoría de Dignaga, sí tenía una postura más cercana a la de Dignaga que a la de Dharmakirti. En 1969, R.S.Y. Chi publicó su estudio sobre la regla trimembre y la rueda de las razones basándose en fuentes tibetanas y chinas. Fue el primer estudio en el mundo occidental que utiliza estos tratados escritos por Dignaga sobre la teoría de la inferencia.

${ }^{13}$ La escuela Nyaya, la principal adversaria de Dignaga, no aceptaba la distinción entre dos tipos de razonamiento. Sólo hasta el siglo XIII, la Navya Nyaya (la nueva Nyaya) la consideró una tesis aceptable. 
(jñapaka-hetu). Esta clasificación dicotómica podría parecer un poco trivial, ya que aparentemente se está refiriendo a los dos lados de la misma moneda. Pero si lo analizamos con más detalle, descubrimos que las dos funciones de la razón no son totalmente iguales, porque representan diferentes procesos cognitivos: el del agente que defiende su tesis y el de su oponente.

A fin de poder realizar exitosamente una argumentación, la razón iluminativa en el lado del agente $p_{1}$ que defiende su tesis tiene un papel causal, cuyo efecto se manifiesta en la razón comprensiva de su oponente $p_{2}$. Un agente, para poder defender su tesis, tiene que haber realizado los siguientes tres pasos cognitivos. Primero, poseer inteligencia suficiente para construir su tesis y las razones que la apoyen; segundo, entender los significados de la tesis y sus razones. Por la palabra "significado", Dignaga y sus comentaristas chinos entienden algo no muy diferente de lo que entiende la mayoría de los filósofos occidentales, algo que tiene dos componentes: el sentido y el referente. El tercer paso cognitivo requiere tener la capacidad de expresar la tesis y las razones a través de un lenguaje comprensible tanto para él mismo como para su oponente. El oponente, por su parte, también tiene que cumplir tres pasos cognitivos para llevar a cabo una argumentación. Primero, tener la capacidad lingüística de distinguir la tesis y las razones en cuestión; segundo, entender los significados de la tesis y sus razones; y tercero, tener la capacidad suficiente para juzgar si es un buen razonamiento o no. Estos seis pasos cognitivos del agente y su oponente son factores imprescindibles para configurar el contexto de una argumentación. Es importante tomar en cuenta que, al especificar las dos funciones de la razón y sus seis pasos cognitivos, Dignaga ubica su teoría del razonamiento dentro de los contextos discursivos en que se realiza una argumentación.

\subsection{La tesis}

Una vez que un agente $p_{1}$ está en la situación de argumentar con su oponente $p_{2}$, el primer paso para realizar un razonamiento trimembre, según Dignaga, es que $p_{1}$ presente la tesis (paksadharma) que va a defender. Un enunciado de la tesis comprende dos partes que concuerdan con el sujeto (dharmin) y el predicado (dharma) en la terminología de la lógica occidental. Por ejemplo, en la tesis "la montaña tiene fuego", "la montaña" es el sujeto y "tiene fuego" es el predicado. Al formular y presentar una tesis, un agente afirma que el sujeto tiene una propiedad expresada por el predicado. En una argumentación, o en un razonamiento del tipo pararthanumana, en que un agente $p_{1}$ defiende una tesis ante su oponente $p_{2}$, una tesis tiene que cumplir los dos requisitos siguientes para que pueda ser una tesis. 
En primer lugar, ambas partes tienen que asentir a lo que el sujeto y el predicado significan. El cumplimiento de este requisito garantiza que una argumentación pueda realizarse en torno a una misma tesis y no se desvíe de ella. Por ejemplo, una persona $A$ ve que hay humo en una montaña y presenta a otra persona $B$ la tesis hipotética "la montaña está envuelta en fuego", pero $B$ no está de acuerdo con lo que $A$ señala. Para $B$, lo que está humeando es una piedra. En este caso, lo que está en discusión ya no es la tesis "la montaña está envuelta en fuego", sino la cuestión de "es una montaña la que está humeando o una piedra". Lo mismo sucede con el predicado en cuestión. $A$ y $B$ tienen que estar de acuerdo con lo que significa la palabra "fuego" para poder establecer una tesis a partir de la cual se puede argumentar.

El segundo requisito para establecer una tesis es que las dos partes de la argumentación no estén de acuerdo en que el sujeto tenga en efecto la propiedad expresada por el predicado. Si $A$ y $B$ están de acuerdo con el hecho de que en la montaña hay fuego, no se va a argumentar sobre esto.

\subsection{La razón}

Una vez que se ha establecido una tesis, el agente $p_{1}$ tiene que presentar una razón para defenderla. Por lo general, la teoría del razonamiento de Dignaga así como las de otras escuelas de la India antigua requieren que la razón se introduzca con la palabra "porque". Por ejemplo, la razón de la tesis "la montaña está en llamas" puede ser "porque tiene humo", en la que no se necesita repetir el sujeto. En este caso, el predicado "tiene humo" es llamado por los filósofos indios antiguos la razón (hetu).${ }^{14}$ Como hemos visto en la sección anterior, Dignaga plantea dos grupos de reglas para garantizar la seguridad de la razón: la regla trimembre (trairupya) y la rueda de las razones (hetu-craka).

Comenzaremos con la regla trimembre, que comprende tres subreglas, la primera de la cuales dice:

(Subregla I) Paksadharmata: El sujeto de la tesis seguramente tiene la propiedad expresada por la razón. ${ }^{15}$

En nuestro caso, para que podamos argumentar a favor de la tesis de que la montaña está en llamas, basándonos en la presencia del humo, tenemos que estar seguros de que el humo que hemos visto es una propiedad de la montaña en ese momento; o sea, tenemos que estar seguros de que el

\footnotetext{
${ }^{14}$ Siguiendo el estilo ortodoxo de la escuela Nyaya, en vez de "hetu", la razón también se escribe como "linga", que significa el signo. Por eso, algunos estudios llaman a la regla trimembre de Dignaga la teoría de los signos (Matilal 1998, pp. 88-107).

${ }^{15}$ Con respecto a las diferentes versiones para expresar la regla trimembre en idioma occidental, véase Chi 1969, pp. 31-35.
} 
humo realmente aparece en la montaña que la tesis refiere. El humo que aparece en otras montañas no servirá como una razón aceptable. En el caso de la montaña incendiada, la razón es la observación de un evento particular. Existen también ejemplos en los que la razón es una propiedad abstracta. Por ejemplo, en la filosofía india antigua se discutía frecuentemente sobre la tesis de que si el sonido es permanente o no. Para argumentar que el sonido no es permanente, un agente presenta la razón "porque (un sonido) es producido", que es una razón que cumple lo que pide la primera subregla de la regla trimembre, porque todos los sonidos tienen la propiedad de ser producidos de una u otra manera. La violación de esta subregla generalmente nos lleva a errores. Por ejemplo, si para defender la tesis de que el sonido no es permanente, alguien presenta una locución "porque es pronunciado por una cuerda vocal" como razón, no es difícil ver que esta razón no es aceptable porque viola la subregla I. Si bien algunos sonidos son pronunciados por una cuerda vocal, existen en la naturaleza otros sonidos que son producidos por otros medios, y para éstos la locución anterior no sirve como razón para apoyar la tesis.

Es preciso mencionar un punto importante destacado por los comentaristas chinos. Como sabemos, en una tesis que se presenta en una argumentación, el sujeto (dharmin) posee la propiedad expresada por el predicado (dharma) de la tesis, pero el agente $p_{1}$ y su oponente $p_{2}$ no deben estar de acuerdo con ello, por lo cual es necesario procesar la argumentación. Para poder establecer una razón, se requiere que el sujeto (dharmin) posea la propiedad expresada por la razón (hetu), pero esta vez tanto el agente $p_{1}$ como su rival $p_{2}$ tienen que estar de acuerdo al respecto para poder continuar el procedimiento de la argumentación. Así, si bien las dos partes en una argumentación tienen que estar en desacuerdo con la idea de que el sujeto tenga la propiedad expresada por el predicado en la tesis, sí deben estarlo en cuanto a la idea de que el sujeto tenga la propiedad expresada por la razón.

Sin embargo, si bien esta subregla exige algo que es necesario para establecer una razón y elimina algunas razones mal planteadas, no puede garantizar que todas las razones ofrecidas sean correctas. Por ejemplo, para defender la tesis de que el sonido no es permanente, uno puede presentar la locución "porque es invisible a la observación directa", que es una locución que no viola la subregla I, puesto que todos los sonidos son invisibles a la observación directa. Pero esta locución tampoco sirve para defender la tesis. Para evitar este tipo de razones mal planteadas, Dignaga plantea las siguientes dos subreglas:

(Subregla II) Sapakse sattva: Existe por lo menos un ejemplo positivo (sapaksa) que tiene la propiedad expresada por el predicado (dharma) de la tesis, y tiene también la propiedad 
expresada por la razón (hetu); además, un ejemplo positivo no puede ser ni el sujeto (dharmin) ni sus casos particulares.

(Subregla III) Vipakse asattva: No existe el caso de que un ejemplo negativo (vipaksa), que no tiene la propiedad expresada por el predicado (dharma) de la tesis, tenga la propiedad expresada por la razón (hetu).

Mientras la subregla I condiciona la relación entre el predicado y la razón de una tesis, estas dos subreglas en conjunto rectifican la relación entre el predicado y la razón de la tesis. La distinción entre un ejemplo positivo y un ejemplo negativo depende de su relación con la propiedad expresada por el predicado de una tesis. Un ejemplo positivo (sapaksa) posee la propiedad expresada por el predicado; además, un ejemplo positivo no puede ser el sujeto o sus casos particulares en la tesis que se defiende. La subregla II requiere que, para que un razonamiento sea bueno, debe existir por lo menos un caso del ejemplo positivo que tenga la propiedad de la razón. En el ejemplo de la montaña incendiada, donde el predicado es "está en llamas" y la razón es "tiene humo", el ejemplo positivo que los filósofos indios antiguos pusieron es una cocina, en la que se ve con frecuencia que el humo se produce por el fuego. Un ejemplo negativo es una cosa que no posee la propiedad del predicado de una tesis, y la subregla III requiere que tanto el agente $p_{1}$ como su rival $p_{2}$ no encuentren un caso en que un ejemplo negativo tenga la propiedad expresada por la razón. En el ejemplo de la montaña con fuego, un ejemplo negativo puede ser un estanque, que no posee la propiedad de tener fuego, la propiedad expresada por el predicado, y que, como la subregla III lo exige, tampoco posee la propiedad expresada por la razón, que es tener humo.

Un punto importante que no hay que olvidar es que la subregla II especifica que el sujeto y sus casos particulares de una tesis no pueden servir como un ejemplo positivo. Dignaga pone este requerimiento por la consideración del contexto de una argumentación. Como hemos visto anteriormente, un requisito para que un agente $p_{1}$ presente una tesis es que su oponente $p_{2}$ no esté de acuerdo con lo que dice la tesis, o sea, que el oponente $p_{2}$ crea que el sujeto de la tesis no tiene la propiedad expresada en el predicado. Para defender su tesis, el agente $p_{1}$ presenta la razón. $\mathrm{Si}$, en el procedimiento para establecer la razón, el agente $p_{1}$ utiliza el sujeto como un ejemplo positivo que sirve como una parte de la razón, simplemente está haciendo una petición de principio, porque decir que el sujeto, o sus casos particulares, es un ejemplo positivo equivale a decir que el sujeto tiene la propiedad expresada por el predicado, que es precisamente lo que dice la tesis que va a defenderse. Como es el caso de que el agente defienda la tesis "la montaña está en llamas" y presente la razón "porque 
tiene humo". Al establecer la razón hay que encontrar un ejemplo positivo, una cosa que tiene la propiedad de estar en llamas, la propiedad expresada por el predicado, y que también tiene humo, la propiedad expresada por la razón. Si el agente $p_{1}$ utiliza la montaña en cuestión como el ejemplo positivo, en la razón que se requiere para justificar la tesis "la montaña tiene fuego", ya está la idea "la montaña tiene fuego", lo que no se permite en una argumentación correcta. Especificando el requisito de que el sujeto de una tesis no debe utilizarse como un ejemplo positivo, la teoría de Dignaga intenta evitar un problema parecido al petitio principii, problema discutido por muchos filósofos occidentales. Sobre ello hablaré con más detalle en la siguiente sección.

En las subreglas II y III, Dignaga especifica la condición que un razonamiento debe cumplir para ser un buen razonamiento, la cual dice que existe por lo menos un ejemplo positivo y ningún ejemplo negativo con la propiedad expresada por la razón. En un estudio descriptivo de las posibles combinaciones de los ejemplos positivos y los negativos, el estudio llamado "la rueda de las razones", Dignaga llega a la misma conclusión. Un ejemplo positivo con respecto a la propiedad expresada por la razón podría tener tres posibilidades: todos los ejemplos positivos la tienen, algunos la tienen y, finalmente, ninguno la tiene. Lo mismo ocurre con los ejemplos negativos: todos los ejemplos negativos tienen la propiedad expresada por la razón, algunos la tienen y ninguno la tiene. De tal forma, existen nueve posibilidades de combinación de los ejemplos positivos y negativos. Las veremos con más detenimiento.

La combinación I. Todos los ejemplos positivos y todos los ejemplos negativos tienen la propiedad expresada por la razón. Por ejemplo, si para defender la tesis "los seres humanos son animales", presento la razón "porque son objeto de los estudios científicos", tenemos un caso de ese tipo. En este caso, los ejemplos positivos son los animales, como los perros y los tigres, todos los cuales pueden ser objeto de estudios científicos; y los ejemplos negativos, que no son animales, por ejemplo las plantas y las minas, también pueden ser objeto de estudios científicos. La razón en este caso no sirve para apoyar la tesis. ${ }^{16}$

La combinación II. Todos los ejemplos positivos tienen la propiedad expresada por la razón y los negativos no. Por ejemplo, la tesis es "los seres humanos son mortales" y la razón es "porque son seres vivos". En este caso, los ejemplos positivos, que son los mortales, tienen la propiedad de

\footnotetext{
${ }^{16}$ Para ilustrar la rueda de las razones, Dignaga utiliza ejemplos tomados de las discusiones entre las diferentes escuelas filosóficas de la India antigua sobre el tema de la naturaleza del sonido. Algunos de ellos pueden ser difíciles de entender para los que no están familiarizados con las tesis principales de estas escuelas. Por ello, he puesto en su lugar otros ejemplos más ordinarios. Para una versión de los ejemplos utilizados por Dignaga, véase Chi 1969, pp. 5-7.
} 
ser o bien los animales o bien las plantas, y todos los ejemplos negativos, que no son mortales, como las piedras y el aire, no tienen la propiedad de ser seres vivos. En esta ocasión, la razón sirve como un buen apoyo para la tesis.

La combinación III. Todos los ejemplos positivos tienen la propiedad expresada por la razón, y algunos ejemplos negativos tienen esa propiedad pero otros no. Por ejemplo, para defender la tesis "los seres humanos son animales" presento la razón "porque son seres vivos". Los ejemplos positivos, que son los animales, tienen la propiedad de ser seres vivos, mientras que algunos ejemplos negativos, que en este caso son las plantas, tienen la propiedad de ser seres vivos; pero otros ejemplos negativos, tales como las piedras y el aire, no tienen dicha propiedad. En esta combinación, la razón no apoya la tesis.

La combinación IV. Ningún ejemplo positivo tiene la propiedad expresada por la razón, y todos los ejemplos negativos tienen esa propiedad. Por ejemplo, para defender la tesis "los seres humanos son inmortales", propongo la razón "porque son seres vivos". Ninguno de los ejemplos positivos, que son todas aquellas cosas que son inmortales, tiene la propiedad de ser un ser vivo; mientras que todos los ejemplos negativos, que son mortales, tienen la propiedad de ser seres vivos. Obviamente, la razón propuesta no sólo no apoya la tesis en cuestión, sino la refuta.

La combinación $V$. Ningún ejemplo positivo tiene la propiedad expresada por la razón, y lo mismo ocurre con los ejemplos negativos. Por ejemplo, para defender la tesis "los seres humanos son seres vivos", propongo la razón "porque son animales racionales". Los ejemplos positivos, que son seres vivos, no tienen la propiedad de ser animales racionales, salvo los seres humanos. Pero, como hemos visto anteriormente, para evitar el caso de que una razón lleve a una petición de principio, el sujeto y sus casos particulares de una tesis no pueden considerarse como ejemplos positivos, no podemos contar en este caso a los seres humanos como ejemplos positivos, de manera que ningún ejemplo positivo tiene la propiedad expresada por la razón. Lo mismo ocurre con los ejemplos negativos, porque una cosa que no es un ser vivo no puede ser un animal racional. Para Dignaga, en esta combinación, la razón propuesta tampoco apoya la tesis. Es importante notar que, para los que están familiarizados con la lógica occidental, este resultado podría parecer extraño, puesto que para un silogismo cuya premisa mayor es "los animales racionales son seres vivos" y cuya premisa menor es "los seres humanos son animales racionales", es totalmente válido concluir que "los seres humanos son seres vivos". Sin embargo, para la teoría del razonamiento de Dignaga, si el agente quiere utilizar en una argumentación el enunciado "los seres humanos son animales racionales" 
como razón para defender la tesis "los seres humanos son seres vivos", está cometiendo una petición de principio, por ello sostiene que este tipo de razón no es una buena razón. A esta diferencia entre la teoría del razonamiento de Dignaga y la lógica occidental me referiré más adelante.

La combinación VI. Ningún ejemplo positivo tiene la propiedad expresada por la razón, mientras que algunos ejemplos negativos tienen esa propiedad pero otros no. Por ejemplo, cuando la tesis es "los seres humanos son inmortales" y la razón propuesta es "porque son animales". En este caso, ningún ejemplo positivo de los inmortales es un animal, mientras que algunos ejemplos negativos que son mortales son animales, pero los otros, como las plantas, no son animales. La razón propuesta en este caso tampoco se considera como buena para apoyar la tesis.

La combinación VII. Algunos ejemplos positivos tienen la propiedad expresada por la razón pero otros no, mientras que todos los ejemplos negativos tienen esa propiedad. Por ejemplo, para defender la tesis "los seres humanos no son animales", se propone la razón "porque son seres vivos". En este caso, algunos ejemplos positivos que no son animales, tales como las plantas, son seres vivos, pero otros ejemplos positivos, tales como las piedras y el aire, no tienen la propiedad de ser seres vivos. Con respecto a los ejemplos negativos, todos los animales son seres vivos. En este caso, la razón propuesta tampoco es buena.

La combinación VIII. Algunos ejemplos positivos tienen la propiedad expresada por la razón pero otros no, mientras que ninguno de los ejemplos negativos tiene dicha propiedad. Por ejemplo, para defender la tesis "los seres humanos son mortales", se propone la razón "porque son animales". Algunos ejemplos positivos que son mortales son animales, pero otros ejemplos positivos, como las plantas, no lo son. Pero ninguno de los ejemplos negativos, o sea, las cosas inmortales, tiene la propiedad de ser un animal. En este caso, la razón propuesta sí apoya la tesis.

La combinación IX. Algunos ejemplos positivos tienen la propiedad expresada por la razón pero otros no, mientras que algunos ejemplos negativos también tienen esa propiedad pero otros no. Por ejemplo, para defender la tesis "los seres humanos son blancos", se propone la razón "porque son animales". En este caso, algunos ejemplos positivos que son cosas blancas son animales, otros ejemplos positivos no; mientras que algunos ejemplos negativos que no son cosas blancas tienen la propiedad de ser animales, otros ejemplos negativos no. En esta situación, la razón propuesta no apoya la tesis.

En resumen, únicamente en la combinación II y VIII podemos tener una buena razón para apoyar la tesis que se defiende. En estas dos situaciones, 
o bien todos o bien algunos de los ejemplos positivos tienen la propiedad expresada por la razón en cuestión, que es precisamente lo que la subregla II exige; mientras que ninguno de los ejemplos negativos tienen esta propiedad, que es lo que la subregla III demanda. De tal manera, la rueda de las razones tiene el mismo resultado que la regla trimembre. La diferencia entre ellas es que, mientras que en la rueda de las razones Dignaga hace una investigación descriptiva acerca de las condiciones en las que una razón puede apoyar una tesis, en la regla trimembre el autor especifica de manera normativa estas condiciones. De acuerdo con Dignaga, un agente presenta apropiadamente una razón cuando ha garantizado que o bien la regla trimembre ha sido satisfecha, o bien cuando se ha cumplido la combinación II o la VIII de la rueda de las razones junto con la subregla I.

\subsection{Los ejemplos}

Una vez que ha presentado una razón, el agente $p_{1}$ tiene que complementar su razonamiento presentando ejemplos, tanto positivos como negativos. La forma de hacerlo es la siguiente: tomando el ejemplo de la montaña en fuego, una vez que se haya asegurado de que la razón "porque tiene humo" es correcta de acuerdo con la regla trimembre o la rueda de las razones, tiene que presentar los ejemplos de la forma: "existe por lo menos un ejemplo positivo en el cual hay humo, que podría ser una cocina; y no existe un caso de ejemplo negativo en el cual haya humo, por ejemplo, un estanque". Es costumbre que los filósofos de la India antigua, incluso los de la escuela Nyaya, presenten únicamente la parte del ejemplo positivo; v.gr., en nuestro caso de la montaña en llamas se dice solamente "por ejemplo, en una cocina". Dignaga permite eso, ya que tanto los ejemplos positivos como los negativos se han examinado en el proceso de establecimiento de la razón, pero destaca que es sólo una abreviación de la forma completa.

\section{Por qué la teoría de Dignaga no puede ser reconstruida por la lógica formal}

Ahora podemos tener un esquema general de la inferencia trimembre de Dignaga, que, en lugar de tener una dirección inferencial que va de la fundamentación a la confirmación y, finalmente, a la tesis, como lo supone Bochenski, tiene una dirección inferencial que va de la tesis a la razón y los ejemplos, de acuerdo con lo que piden los contextos prácticos de una argumentación. Escribimos este esquema tomando el ejemplo de la montaña en llamas:

La tesis: La montaña está en llamas.

La razón: Porque tiene humo. 
Los ejemplos: Existe por lo menos un ejemplo positivo en el cual hay humo, como en una cocina; y no existe un ejemplo negativo en el cual haya humo, como en un estanque.

Para realizar la inferencia expresada por el esquema, el agente $p_{1}$ tiene que dar los siguientes pasos. En primer lugar, presentar la tesis que es la montaña que está en llamas. Una vez que su rival $p_{2}$ haya expresado la objeción o la duda acerca de la tesis, el agente $p_{1}$ presenta la razón que es "porque tiene humo". Para establecer esta razón, el agente $p_{1}$ tiene que averiguar si la razón propuesta cumple las tres subreglas de la regla trimembre, o sea, tiene que examinar si la razón propuesta es una propiedad del sujeto de la tesis, si existe un ejemplo positivo que tenga la propiedad expresada por la razón y si ninguno de los ejemplos negativos que puedan ser imaginados tiene esa propiedad. Cuando ha garantizado mentalmente que la razón cumple todos estos requisitos, presenta la razón y los ejemplos. Por su parte, el oponente $p_{2}$, después de enterarse de la razón presentada por el agente $p_{1}$, realiza en su mente los mismos procedimientos cognitivos para averiguar si la razón propuesta cumple la regla trimembre. Una vez que se ha asegurado de que los cumple, tiene que aceptar que la tesis está bien apoyada por la razón.

Al comparar la teoría del razonamiento de Dignaga con las teorías del razonamiento occidentales que dan por sentada la propuesta formalista, podemos encontrar las siguientes características de la teoría de Dignaga que no pueden reconstruirse con la lógica formal.

La primera es que, a diferencia de la propuesta formalista, en la que las consideraciones sobre los factores contextuales de un razonamiento han sido disminuidas a un nivel mínimo, en la teoría de Dignaga, los principios normativos del razonamiento siempre se dan dentro de los contextos prácticos en los que se realiza una argumentación. Dignaga distingue el razonamiento que un agente hace para sí mismo (svabhavanumana) del razonamiento para argumentar con los otros (pararthanumana), y, como hemos visto en $2.1,2.2$ y 2.3 , en este último modo de razonamiento, los requisitos normativos en cada paso de la inferencia tienen que ver con el contexto en que un agente $p_{1}$ está argumentando con su rival $p_{2}$. Por ejemplo, para que el agente $p_{1}$ pueda presentar una tesis, las dos partes involucradas en la argumentación deberán estar de acuerdo con el significado del sujeto y el predicado de la tesis, pero divergir con respecto a la propiedad del sujeto que expresa el predicado. Mientras que en el procedimiento mediante el que se establece una razón, ambas partes de la argumentación tienen que estar de acuerdo con el requisito de la regla trimembre.

La segunda característica especial de la teoría de Dignaga es la dirección inferencial. Aquí me gustaría profundizar con más detalle en la cues- 
tión sobre la dirección inferencial de un razonamiento. Un razonamiento puede ser una inferencia que se hace a partir de un conjunto de premisas para llegar a una conclusión. Esto tiene lugar cuando a partir de una proposición probamos sus consecuencias lógicas. En este caso, la dirección inferencial va de las premisas a la conclusión. Un razonamiento también puede ser una inferencia que va de una conclusión a las razones que la apoyan. Un ejemplo es si yo explico a una persona por qué creo que los Pumas ganarán el partido de fútbol del próximo domingo. En esta ocasión, la dirección inferencial puede ir de la conclusión, la cual afirma que los Pumas ganarán, a las premisas que son ciertas razones que apoyan esta conclusión. Una objeción directa a esta distinción de dos tipos de razonamiento dependiente de la dirección inferencial diría que no es una clasificación útil para nuestro entendimiento acerca del razonamiento humano, ya que, en la lógica, la relación inferencial entre dos proposiciones se puede entender en ambas direcciones; esto es, la relación lógica entre dos proposiciones $A$ y $B$ se puede entender tanto de $A$ a $B$ como de $B$ a $A$; de tal modo que, tanto el razonamiento cuya dirección inferencial va de la premisa a la conclusión como el razonamiento cuya dirección inferencial va de la conclusión a la premisa, se puedan reconstruir por la misma forma lógica. Esta objeción presupone ambas tesis: la de la propuesta formalista del razonamiento y la tesis antipsicologista de la lógica. Esta última sostiene que las reglas lógicas no son "leyes psicológicas", en el sentido de que los estudios lógicos se dedican únicamente a cuestiones sobre las reglas normativas, en las que la validez de una inferencia se garantiza; por tal motivo, no tienen nada que ver con los factores psicológicos del pensamiento. La dirección inferencial es una cuestión que tiene que ver con procedimientos y órdenes psicológicos, o quizás epistemológicos, de un razonamiento, pero no es relevante para los estudios lógicos. La tesis antipsicologista en la lógica puede ser correcta, ya que la lógica, como una disciplina independiente, puede definir su propio campo de estudio sin que le preocupen los temas de otras disciplinas. Pero, para poder llegar de esta tesis antipsicologista en la lógica a la conclusión de la tesis antipsicologista en el razonamiento, que sostiene que la dirección inferencial no es una cuestión útil para entender el razonamiento humano, se requiere presuponer otra tesis que afirma que los estudios lógicos nos ofrecen todo lo que necesitamos para entender el razonamiento humano. Esto significa que, por un lado, el razonamiento radica en razonar de acuerdo con las reglas lógicas, y que, por otro, las reglas lógicas representan correctamente los principios normativos del razonamiento. Obviamente, esta tesis es precisamente lo que la propuesta formalista del razonamiento sostiene. Ahora podemos resumir, de la siguiente manera, el argumento que se opone a la importancia de la dirección inferencial: 
(1) El problema filosófico del razonamiento reside únicamente en analizar la relación lógica y probabilística entre las premisas y la conclusión, y no en otros factores (tesis de la propuesta formalista del razonamiento).

(2) El estudio de la lógica se dedica exclusivamente a cuestiones sobre la validez de una inferencia y no tiene nada que ver con los factores psicológicos de un razonamiento (tesis antipsicologista).

(3) La dirección inferencial es una cuestión que tiene que ver con procedimientos y órdenes psicológicos.

(Conclusión) Por lo tanto, la cuestión de la dirección inferencial no es un problema filosófico del razonamiento.

Como hemos visto anteriormente, lo que la premisa (3) dice es un hecho y la premisa (2) tampoco es problemática. El problema se concentra en la premisa (1), que es la propuesta formalista del razonamiento. De acuerdo con los nuevos proyectos del razonamiento que rechazan la propuesta formalista, proyectos que hemos visto en la parte introductoria, para que las reglas lógicas puedan representar correctamente los principios normativos del razonamiento tienen que adecuarse a factores contextuales, como factores psicológicos y sociales, entre otros.

La dirección inferencial es uno de estos factores que configuran los contextos de un razonamiento. Michael Dummett ha hecho notar este punto en su artículo "La justificación de la deducción" (1973). Dummett distingue dos tipos de razonamiento: el argumento persuasivo y el argumento explicativo, de acuerdo con su función pragmática. Un argumento persuasivo es un razonamiento cuya realización consiste en persuadir a alguien, o quizá a nosotros mismos, de que una conclusión es correcta; mientras que, en un argumento explicativo, queremos explorar las razones para apoyar una conclusión que ya hemos considerado correcta. Como argumenta el autor, estos dos tipos de razonamiento tienen diferentes direcciones inferenciales, debido a que sus tareas pragmáticas y epistemológicas son diferentes:

Una explicación con frecuencia toma la forma de la construcción de un argumento deductivo, cuya conclusión es un enunciado del hecho que necesita explicarse: pero, a diferencia de lo que pasa en un argumento persuasivo, en un argumento explicativo la dirección epistémica es inversa a la dirección de la inferencia lógica. En un argumento persuasivo, la dirección epistémica debe coincidir con la secuencial: es necesario que las premisas del argumento sean proposiciones que ya sean consideradas verdaderas por la persona a la cual queremos persuadir de la verdad de la conclusión. De manera característica, en una explicación, la conclusión del argumento está dada de antemano; y muy bien podría suceder que nuestra única razón para creer en las premisas 
del argumento explicativo fuera que proporcionan la explicación más verosímil de la verdad de la conclusión. (Dummett 1973, p. 383)

Aquí Dummett distingue dos tipos de direcciones en un razonamiento. En primer lugar, con la expresión "la dirección de la inferencia lógica", Dummett se refiere a un concepto de la relación lógica entre premisa y conclusión, y toma la dirección de la premisa a la conclusión como dirección normal; pero, como hemos visto antes, la validez de una inferencia se entiende en ambas direcciones: la de las premisas a la conclusión y la de la conclusión a las premisas. Podemos decir que este tipo de dirección de un razonamiento es un concepto lógico que no tiene que ver con factores psicológicos ni con otros factores contextuales. En segundo lugar, Dummett introduce el concepto de dirección epistémica que es relevante para los factores psicológicos y epistémicos. Lo que distingue un argumento persuasivo de otro explicativo es que la dirección epistémica del primero es inversa a la del segundo. Esto es, en un argumento persuasivo inferimos una conclusión a partir de un conjunto de premisas consideradas verdaderas; mientras que en un argumento explicativo inferimos, a partir de la conclusión considerada verdadera, las razones que la apoyan y que, por lo tanto, la explican. Al introducir esta distinción entre el argumento persuasivo y el argumento explicativo, la intención de Dummett es argüir que el método para justificar la deducción —un problema filosófico que no ha sido resuelto satisfactoriamente hasta hoy en día- debería residir en el argumento explicativo, porque, según el autor, es la única manera de evitar la acusación de circularidad, o de petición de principio, para esta justificación. No voy a discutir aquí la plausibilidad de este interesante planteamiento. ${ }^{17}$ Lo que me importa aquí es señalar que la introducción de Dummett del concepto de la dirección epistémica nos ofrece un buen ejemplo de por qué es importante hablar sobre la dirección inferencial de un razonamiento, sobre todo cuando las distintas direcciones inferenciales implican diferentes pro-

${ }^{17} \mathrm{El}$ argumento general de Dummett es que el problema de la justificación de la deducción se plantea en tres niveles. El primero es "el caso en el cual un argumento puede validarse construyendo una prueba, de varios pasos, de sus premisas a su conclusión mediante el uso de las formas más simples de inferencia que admitimos como válidas. El segundo nivel es el que también hemos considerado, donde la corrección de una sola forma de inferencia básica, o de toda la sistematización de una cierta área de la lógica, esté en duda: y es en este nivel donde la prueba de la corrección o de la completud semántica pretende al menos proporcionar una justificación. Pero aún hay un tercer nivel, más profundo: es el nivel en el cual pedimos una explicación no de por qué debemos aceptar ciertas formas de argumentos o cánones para juzgar las formas de argumentos, sino de cómo es posible el argumento deductivo" (Dummett 1973, p. 384). El problema es que, en los dos primeros niveles, la justificación es circular, ya que la justificación misma utiliza reglas deductivas. Este círculo desaparece si localizamos la justificación en el tercer nivel, donde la dirección inferencial epistémica va de la conclusión a las premisas. Para una discusión acerca de la plausibilidad de este planteamiento, véase Haack 1982. 
cedimientos epistémicos, psicológicos y pragmáticos. Según Dummett, un argumento persuasivo es un tipo de inferencia diferente de un argumento explicativo, porque ambos tienen diferentes direcciones inferenciales que cumplen diferentes tareas pragmáticas. $\mathrm{O}$, dicho de otra manera, en diferentes contextos y con diferentes tareas pragmáticas, de acuerdo con la diferencia en la dirección inferencial, se pueden clasificar los diferentes tipos de razonamiento.

Volvamos ahora a nuestro caso. Si bien en una lógica formal la cuestión de la dirección inferencial no tiene sentido, para la teoría de Dignaga sí es importante. En la teoría de Dignaga, el agente primero presenta una tesis, que equivale a la conclusión de una inferencia, y luego busca una razón que la apoye. Durante el procedimiento para establecer la razón, el agente examina la relación de apoyo entre la razón y la tesis averiguando los ejemplos positivos y los negativos. Lo que es importante enfatizar aquí es el hecho de que el criterio para decidir si un ejemplo es positivo o negativo depende de si el ejemplo tiene la propiedad expresada por el predicado de la tesis que se defiende. Así, en cada paso cognitivo de la teoría de Dignaga - en la presentación de una tesis, de su razón y de los ejemplos-, el agente tiene que examinar el contenido semántico del sujeto y el predicado de la tesis, y su relación semántica y pragmática con la razón.

La tercera característica de la teoría de Dignaga es que es muy diferente del silogismo occidental. Como ya lo mencionamos, casi todos los intentos de los filósofos occidentales por reconstruir la antigua lógica india tratan de reconstruir o comparar la teoría de Dignaga con el silogismo aristotélico, sobre todo con el modo de Barbara. Aquí son la subregla II y la III las que desempeñan el papel importante de la reconstrucción o la comparación. Tanto Bochenski como Stcherbatsky consideran que, por medio de estas dos subreglas, se puede construir una proposición que equivale a la premisa mayor de un silogismo, en el que la relación entre el término medio y el término mayor se expresan mediante una proposición universal. El truco es que cuando la subregla II dice que existe por lo menos un ejemplo positivo que tiene la propiedad expresada por la razón, y la subregla III dice que ningún ejemplo negativo tiene esa propiedad, los filósofos occidentales piensan que estas dos subreglas en conjunto equivalen a decir que todos los que tienen la propiedad expresada por la razón tienen la propiedad expresada por el predicado de la tesis, lo cual equivale a la premisa mayor de un silogismo aristotélico, que dice que todas las propiedades del término medio están en las propiedades del término mayor. Por ejemplo, en el caso de la montaña en llamas, cuando de acuerdo con las subreglas II y III, el agente dice que existe por lo menos un ejemplo positivo en el que cuando hay fuego hay humo, y que no existe un ejemplo negativo en el que cuando no hay fuego haya humo, lógicamente equivale a decir que en todos los casos en los que hay humo hay 
fuego, lo cual es la premisa mayor de un silogismo que se sigue de la premisa menor "la montaña tiene humo" y la conclusión "la montaña está en llamas".

Sin embargo, esta manera directa de reconstrucción o comparación no es plausible por las siguientes tres razones. En primer lugar, la reconstrucción trivializa la subregla II de la regla trimembre de la teoría de Dignaga. La subregla II es una proposición existencial, que dice que existe un ejemplo positivo que tiene la propiedad expresada por la razón; por eso, a partir de ella no se puede llegar a una proposición universal que diga que todas las propiedades expresadas por la razón (el término medio) están en las propiedades expresadas por el predicado (el término mayor), como lo requiere un silogismo aristotélico. Ahora bien, lo que aparentemente puede llegar a ser una premisa mayor del silogismo es la subregla III, que es una proposición universal, al expresar que no existe un ejemplo negativo en que el que no se tenga la propiedad expresada por el predicado pero se tenga la propiedad expresada por la razón. Esta proposición puede interpretarse utilizando las terminologías del silogismo aristotélico del siguiente modo: no existe un caso en el que los que tengan la propiedad expresada por el término mayor $(\mathrm{P})$ tengan la propiedad expresada por el término medio (M):

$$
-(\exists x)(-\mathrm{P} x \& \mathrm{M} x)
$$

que es lógicamente equivalente a:

$$
(\forall x)(-\mathrm{P} x \rightarrow-\mathrm{M} x)
$$

que es lógicamente equivalente a:

$$
(\forall x)(\mathrm{M} x \rightarrow \mathrm{P} x)
$$

que podría ser la premisa mayor requerida por el silogismo aristotélico. Si esta reconstrucción es correcta, se sigue que la subregla III junto con la subregla I son suficientes para tener una inferencia válida en la cual la tesis está apoyada por la razón, de manera que la subregla II resulta ser innecesaria. Sin embargo, la subregla II no es nada trivial o innecesaria para la consistencia de la teoría de Dignaga, sino que cumple un papel esencial y necesario para asegurar que el agente no cometa cierto tipo de errores en el establecimiento de una razón. Además, tampoco es plausible convertir la subregla III en una premisa mayor de un silogismo, porque tanto la subregla II como la III son resultado de los procesos cognitivos inductivos y falibles que se realizan en la mente de un agente y su rival.

La segunda razón por la que la reconstrucción directa de la teoría del razonamiento de Dignaga mediante el silogismo occidental no es plausible es que, en la teoría de Dignaga, la justificación o el establecimiento de 
una razón depende de un proceso cognitivo y epistémico que ocurre en la mente del agente $p_{1}$ y de su rival $p_{2}$ por medio de una investigación inductiva acerca de los ejemplos positivos y los negativos. Durante este proceso cognitivo y epistémico existen por lo menos dos caracteres que son muy diferentes de un silogismo occidental. Por un lado, el uso de manera esencial de los ejemplos positivos y los negativos significa que la teoría del razonamiento de Dignaga tiene un carácter de inferencia analógica que no se acomoda a una inferencia de silogismo. Por otro lado, los procesos de examinación de los ejemplos positivos y los negativos son esencialmente inductivos. Recordemos que, en la sección anterior, Bochenski califica la teoría del razonamiento de la escuela Nyaya como una "deducción por analogía" por el uso de ejemplos positivos y negativos. Esta calificación no puede aplicarse a la teoría de Dignaga. En la epistemología de Dignaga, que es de tipo antifundamentalista, los universales no son conocimientos innatos, sino que provienen de la abstracción de las experiencias particulares a través de las inferencias inductivas y analógicas. Desde el punto de vista de este tipo de epistemología, el establecimiento de la razón de una tesis mediante el examen de los ejemplos positivos y los negativos debe ser un proceso cognitivo inductivo y falible realizado en la mente de un agente y de su rival. De tal manera, en vez de decir que "existe por lo menos un ejemplo positivo que tiene la propiedad expresada por la razón y no existe ningún ejemplo negativo que tenga esa propiedad", en la teoría de Dignaga se dice, en la mente del agente $p_{1}$ y de su oponente $p_{2}$ : "de acuerdo con mi experiencia existe por lo menos un ejemplo positivo que tiene la propiedad expresada por la razón, y hasta este momento no he encontrado ningún caso en que un ejemplo negativo tenga esa propiedad". Así, no es plausible el intento de identificar la subregla III con la premisa mayor de un silogismo aristotélico, porque en la subregla III tanto el agente como su oponente están diciendo que "hasta el momento no he encontrado un ejemplo negativo que tenga esa propiedad", que es un resultado epistémico inductivo y falible.

La tercera razón por la que la teoría de Dignaga no es directamente reconstruible mediante el silogismo occidental es que, debido al requisito de la subregla II, el sujeto o los casos particulares de una tesis que se defiende no deben considerarse como ejemplo positivo. Esto quiere decir que algunas inferencias válidas de un silogismo pueden considerarse una falacia en la teoría de Dignaga. Por ejemplo, la siguiente inferencia es un silogismo válido:

Todos los animales racionales son seres vivos;

Los seres humanos son animales racionales;

Por lo tanto, los seres humanos son seres vivos. 
Pero, en la teoría del razonamiento de Dignaga, si un agente quiere defender la tesis de que "los seres humanos son seres vivos" presentando la razón "porque son animales racionales", simplemente no puede aducirla porque le falta un ejemplo positivo que tenga la propiedad expresada por la razón. La única clase de ejemplos positivos (ser seres vivos) que tiene la propiedad de la razón (ser animales racionales) es la de los seres humanos. Pero, como hemos visto, la subregla II prohíbe que se considere el sujeto y sus casos particulares, que en nuestro caso es la clase de los seres humanos o sus casos particulares, como un ejemplo positivo; por lo tanto, esta inferencia es una falacia de acuerdo con la teoría de Dignaga, pues carece de un ejemplo positivo. Como mencioné ya, la razón de esta prohibición es evitar una falacia parecida a la de petitio principii, en la que un razonamiento recurre a la conclusión que está por establecerse para justificar esa misma conclusión. Si se permitiera que, para establecer una razón, se utilizaran como ejemplos positivos el sujeto de la tesis que se defiende y sus casos particulares, se estaría permitiendo la afirmación de que el sujeto tiene la propiedad expresada por el predicado, que es precisamente lo que la tesis afirma. En nuestro caso, si para establecer la razón "porque son los animales racionales" se permite que se utilice la clase de los seres humanos, o sus casos particulares, tales como Platón o Aristóteles, como ejemplos positivos (que tienen la propiedad de ser seres vivos) que poseen la propiedad expresada por la razón (los animales racionales), se está afirmando precisamente la tesis que se defiende, a saber, "los seres humanos son seres vivos" para justificar la misma tesis.

Para poder ver este punto con mayor claridad, examinemos brevemente cómo los filósofos occidentales discuten el problema de petitio principii en un silogismo. Fue John Stuart Mill el que afirmó de manera clara que todos los silogismos son esencialmente petitio principii. El ejemplo es la siguiente inferencia silogística:

Todos los seres humanos son mortales,

Sócrates es un ser humano,

Por lo tanto, Sócrates es mortal. (Mill, 1874/1973, p. 184)

Para apoyar la conclusión "Sócrates es mortal", recurrimos a la premisa "todos los seres humanos son mortales". Pero para establecer esta premisa, tenemos que presuponer la conclusión que está por justificarse. Así, para Mill es una petitio principii.

La acusación de que todos los silogismos son petitio principii, como lo hace Mill, es demasiado fuerte. Muchos filósofos han señalado que en muchos usos prácticos, el silogismo puede ser totalmente correcto sin cometer la falacia de petitio principii; por ejemplo, en algunos casos de explicación. Como dice Scarre: 
[L]os silogismos suelen desempeñar un papel explicativo, y sus premisas no sirven para persuadir a alguien de la verdad de una conclusión que haya rechazado antes, sino que ofrecen una razón de por qué es verdadera una conclusión ya conocida. Por ejemplo, podría darme perfecta cuenta de que el pequeño Juan está engordando, pero quiero saber por qué. Alguien que me plantee el siguiente silogismo:

Todos los bebés que comen alimentos marca Crecimax engordan,

Juanito come alimentos Crecimax, por lo tanto,

Juanito está engordando

no me estará tratando de convencer de que Juanito está engordando, puesto que eso ya lo sé; pero me aclarará la causa de que esté engordando. (Scarre 1989, pp. 51-52)

En este caso, la conclusión de que el niño está gordo se ha aceptado epistémicamente como verdadera en la inferencia explicativa; por eso, al aplicar la premisa mayor para explicarla no se tendrá el problema de petitio principii. Sin embargo, tanto Scarre como Dummett (1973) reconocen que en un caso de argumentación persuasiva (persuasive argument), el silogismo sí comete la falacia. Si un agente quiere defender la tesis de que "Sócrates es mortal" y recurre tanto a la premisa menor "porque Sócrates es un ser humano" como a la mayor "todos los seres humanos son mortales", su rival puede desafiar la premisa mayor preguntando: "Antes de que estés seguro de que Sócrates es mortal, ¿cómo puedes saber que todos los seres humanos son mortales?" La teoría del razonamiento de Dignaga, y sobre todo la teoría del razonamiento persuasivo (pararthanumana), tiene un contexto práctico parecido al de este caso de argumentación persuasiva. ${ }^{18}$ Nótese que la dirección inferencial de una argumentación persuasiva es diferente de la de una argumentación explicativa. Hemos visto en el caso de Dummett (1973) que diferentes direcciones inferenciales implican diferentes procesos epistémicos y psicológicos. La dirección epistémica planteada por Dummett refiere a un orden de justificación de las proposiciones en una inferencia; esto es, mientras que en una argumentación persuasiva tenemos que justificar primero las premisas para luego justificar la conclusión, en una argumentación explicativa tenemos primero la conclusión ya justificada y recurrimos a las premisas para explicarla. La dirección lógica se

18 Recordemos que Gilbert Harman (1973, cap. 2) clasifica los tres principales tipos de razonamiento de acuerdo con el contexto pragmático de cada uno. Es interesante notar que estos tres tipos a grandes rasgos corresponden a los diferentes tipos de razonamiento de los que hablamos en este capítulo: el razonamiento para uno mismo (svabhavanumana), el razonamiento para persuadir a los otros (pararthanumana) y, finalmente, el razonamiento para la explicación. 
refiere a la relación lógica entre algunas premisas y su conclusión. Es interesante ver que la dirección epistémica de una argumentación persuasiva, según Dummett, va de la premisa a la conclusión, pero que la dirección inferencial de la argumentación persuasiva, de la que hablo aquí, es al revés. La diferencia es que Dummett toma el silogismo como ejemplo de una argumentación persuasiva, en la que el agente $p_{1}$ tiene que utilizar las premisas para justificar la conclusión, un acto considerado implausible tanto por Dummett como por Dignaga, ya que no se puede evitar la falacia de la petición de principio. Según Dignaga, en una argumentación persuasiva, la inferencia es fundamentalmente inductiva y analógica, su dirección inferencial va de la tesis a las razones que la apoyan, de tal manera que no existe la falacia de la petición de principio, ya que no es una inferencia silogística. Es importante notar que la cuestión de la dirección inferencial y de los contextos prácticos no es menos importante que la de una regla lógica. Un silogismo lógicamente válido, aunque infiere correctamente a partir de un conjunto de premisas una conclusión en una argumentación explicativa, puede ser una falacia en una argumentación persuasiva. Esto quiere decir que mientras que una inferencia es correcta en un contexto práctico y en una dirección inferencial determinada, puede ser una falacia en otros contextos prácticos y en otra dirección inferencial.

El requisito de la subregla II de que el sujeto y sus casos particulares no pueden considerarse ejemplos positivos, como hemos visto, se hace precisamente para evitar la falacia de petitio principii. Para defender la tesis de que "Sócrates es mortal", un agente que adopta la teoría del razonamiento de Dignaga tendrá una estrategia diferente de la que tiene un silogismo. Después de que haya presentado tal tesis, el agente tratará de establecer la razón "porque es un ser humano" a través de los siguientes pasos cognitivos: existe un ejemplo positivo (tiene la propiedad de ser mortal) que tiene la propiedad expresada por la razón (que es un ser humano), por ejemplo, Platón; mientras tanto, hasta este momento no he encontrado ningún caso de ejemplo negativo (que es inmortal) que pueda tener esa propiedad de ser un ser humano, por ejemplo, una piedra (que es inmortal pero no es un ser humano). En esta defensa de la tesis en cuestión, en vez de recurrir a una premisa de forma universal, como ocurre en el silogismo, el agente en la teoría de Dignaga realiza en su mente ciertos procesos cognitivos, inductivos y falibles meditando sobre la relación entre los ejemplos positivos y negativos con la propiedad expresada por la razón.

Así, pues, espero haber mostrado que la teoría del razonamiento de Dignaga es esencialmente diferente de la del silogismo, tan diferente que no existe una reconstrucción directa de la teoría de Dignaga por medio de la teoría del silogismo. Los intentos de los historiadores y los filósofos occidentales por reconstruir la teoría del razonamiento de Dignaga por medio del silogismo se fundamentan en la propuesta formalista, en la que se han 
pasado por alto factores contextuales de la aplicación de un razonamiento. Debido precisamente a esta idea no contextualista del razonamiento, la reconstrucción de la teoría de Dignaga por una lógica formal occidental distorsiona gravemente la idea original de Dignaga.

\section{Conclusión}

En este trabajo, mediante el estudio de un caso histórico, he tratado de demostrar que la propuesta formalista del razonamiento no es plausible, porque el problema de la corrección o incorrección de un razonamiento no sólo depende de las reglas lógicas, sino también de los contextos prácticos en que se realiza este razonamiento. A una teoría del razonamiento no le concierne sólo estudiar la validez lógica de un razonamiento; también tiene que tomar en cuenta los contextos prácticos. La teoría del razonamiento de Dignaga es una teoría que intenta estudiar la validez de un razonamiento en sus contextos prácticos. Dignaga señala que los razonamientos utilizados en una argumentación para persuadir a los que sostienen diferentes puntos de vista pueden ser inferencias de carácter inductivo, cuya dirección inferencial va de una tesis que se defiende a las razones que la apoyan. Para caracterizar este tipo de razonamiento de manera adecuada se tiene que enmarcar en los contextos discursivos de una argumentación, en los que un agente argumenta frente a su rival, como Dignaga lo hace. Si reconstruimos este tipo de razonamiento por medio de una reducción a una lógica formal, si bien esta reconstrucción técnicamente no tendrá problemas, lo haremos al precio de sacrificar consideraciones sobre los contextos prácticos en que se realiza este tipo de razonamiento. La omisión de considerar estos contextos prácticos produce distorsiones o consecuencias paradójicas en nuestro entendimiento de la naturaleza de este tipo de razonamiento, como ocurre en las diferentes maniobras de reconstrucción de la teoría del razonamiento de Dignaga mediante su reducción a un tipo de lógica formal occidental. El fracaso de estas maniobras no debe parecer extraño si aceptamos la tesis de que un entendimiento adecuado de los razonamientos humanos debe tomar en cuenta sus contextos prácticos.

\section{BIBLIOGRAFÍA}

Barlingay, S.S., 1965, A Modern Introduction to Indian Logic, National Publishing House, Delhi.

Barnes, B., 1977, Interests and the Growth of Knowledge, Routledge and Kegan Paul, Londres.

__ 1974, Scientific Knowledge and Sociological Theory, Routledge and Kegan Paul, Londres. 
Bhatt, S.R., 1997, "Logic and Language in Buddihism", en B. Carr e I. Mahalingam (comps.) Companion Encyclopedia of Asian Philosophy, Routledge, Londres, pp. 414-434.

Bhattacharya, V. (comp.), 1927, Nyayapravesa Part II, Gaekwad's Oriental Books, Baroda.

Biagioli, M., 1993, Galileo Courtier-The Practice of Science in the Culture of Absolutism, The University of Chicago Press, Chicago.

Bijker, W.E., T.P. Hughes y T.J. Pinch, 1987, The Social Construction of Technological Systems, The MIT Press, Cambridge.

Bloor, D., 1976/1991, Knowledge and Social Imagery, Routledge and Kegan Paul, Londres (2a. ed.: 1991, The University of Chicago Press, Chicago).

Bochenski, I.M., 1956, Formale Logik, Verlag Karl Alber, Friburgo/Munich. [Versión en castellano: Historia de la lógica formal, trad. Millán Bravo Lozano, Gredos, Madrid, 1985.]

Braine, M.D.S. y D.P. O’Brien, 1991, "A Theory of If: A Lexical Entry, Reasoning Program, and Pragmatic Principles", Psychological Review, vol. 98, pp. 182-203.

Brown, H., 1988, Rationality, Routledge, Nueva York.

Chattopadhyaya, D. (comp.), 1978, Studies in the History of Indian Philosophy-An Anthology of Articles by Scholars, Eastern and Western, K.P. Bagchi and Company, Calcuta, 3 vols.

Chattopadhyaya, D. y M. Gangopadhyaya, 1967, Nyaya Philosophy-Literal Translation of Gautama's Nyaua-sutra \& Vatsyayana's Bhasya along with a Free and Abridged Translation of the Elucidation by Mahamahopadhyaya Phanibhusana Tarkavagisa (Part 1: First Adhyaya), Indian Studies Past and Present, Calcuta.

Chi, R.S.Y, 1969, Buddhist Formal Logic (Part I)—A Study of Dignaga's Hetucakra and K'uei-chi's Great Commentary on the Nyayapravesa, The Royal Asiatic Society of Great Britain and Ireland, Londres.

Collins, H., 1985, Changing Order: Replication and Induction in Scientific Practice, Sage, Londres/Beverly Hills.

Dasgupta, S.M.A., 1922, A History of Indian Philosophy, Cambridge University Press, Cambridge, vol. 1.

Dhruva, M. (comp.), 1930, The Nyayapravesa Part I, Gaekwad's Oriental Series, Baroda.

Dummett, M.A.E., 1973, “The Justification of Deduction”, British Academy Lecture, compilado en Truth and Other Enigmas, Duckworth, Londres, 1978, pp. 290318. [Versión en castellano: "La justificación de la deducción", en La verdad y otros enigmas, Fondo de Cultura y Económica, México, 1990, pp. 377-407. Se cita de esta última.]

Evans, J.St.B., S.E. Newstead y R.M.J. Byrne, 1993, Human Reasoning: The Psychology of Deduction, Erlbaum, Hove.

Evans, J.St.B. y D.E. Over, 1996, Rationality and Reasoning, Erlbaum, Hove.

Franklin, A., 1990, Experiment, Right and Wrong, Cambridge University Press, Cambridge.

— 1986, The Neglect of Experiment, Cambridge University Press, Cambridge.

Fuller, S., 1989, Philosophy of Science and its Discontents, Westview Press, Boulder.

Galison, P., 1997, Image and Logic-A Material Culture of Microphysics, The University of Chicago Press, Chicago. 
Galison, P., 1987, How Experiments End, The University of Chicago Press, Chicago. Giere, R.G., 1988, Explaining Science-A Cognitive Approach, The University of Chicago Press, Chicago.

Gooding, D., 1990, Experiment and the Making of Meaning-Human Agency in Scientific Observation and Experiment, Kluwer Academic Publishers, Dordrecht.

Gooding, D., T.J. Pinch y S. Schaffer (comps.), 1989, The Uses of Experiment: Studies of Experimentation in the Natural Science, Cambridge University Press, Cambridge.

Haack, S., 1982, "Dummett's Justification of Deduction", Mind, vol. XCI, pp. 216239.

Hacking, I., 1983, Representing and Intervening, Cambridge University Press, Cambridge. [Versión en castellano: Representar e intervenir, trad. Sergio F. Martínez, Instituto de Investigaciones Filosóficas-UNAM/Paidós, 1996.]

Harman, G., 1973, Thought, Princeton University Press, Princeton.

Henle, M., 1962, "On the Relation Between Logic and Thinking", Psychological Review, vol. 69, pp. 366-378.

Hutchins, E., 1995, Cognition in the Wild, The MIT Press, Cambridge, Mass.

Inhelder, B. y J. Piaget, 1958, The Growth of Logical Thinking from Children to Adolescence, Basic Books, Nueva York.

Junankar, N.S., 1978, Gautama: The Nyaya Philosophy, Motilal Banarsidass, Delhi.

Kahneman, D., P. Slovic y A. Tversky (comps.), 1982, Judgement under Uncertainty: Heuristics and Biases, Cambridge University Press, Cambridge.

Kalupahana, D.J., 1992, A History of Buddist Philosophy—Continuities and Discontinuities, University of Hawaii Press, Honolulu.

Kitcher, P., 1993, The Advancement of Science, Oxford University Press, Nueva York. [Versión en castellano: El avance de la ciencia, trad. Hector Islas y Laura Manríquez, revisión técnica de la trad. Carlos López Beltrán, Instituto de Investigaciones Filosóficas-UnAm, México, 2001.]

Kneale, W. y M. Kneale, 1962, The Development of Logic, Clarendon, Oxford.

Latour, B., 1987, Science in Action, Open University Press, Milton Keynes.

Lukasiewicz, J., 1957, Aristotle's Syllogistic from the Standpoint of Modern Formal Logic, 2a. ed., Clarendon, Oxford.

Manktelow, K.L. y D.E. Over, 1993, Rationality: Psychological and Philosophical Perspectives, Routledge, Londres/Nueva York.

- (comps.), 1990, Inference and Understanding: A Philosophical and Psychological Perspective, Routledge, Londres.

Matilal, B.K., 1998, The Character of Logic in India, State University of New York Press, Nueva York.

McCulloch, W.S. y W.H. Pitts, 1943, "A Logical Calculus of the Ideas Immanent in Nervous Activity", The Bulletin of Mathematical Biophysics, vol. 5, pp. 115-133.

Mill, J.S., 1874/1970, A System of Logic Ratiocinative and Inductive-Being a Connected View of Principles of Evidence and the Methods of Scientific Investigation, University of Toronto Press/Routledge and Kegan Paul, Toronto/Londres.

Mookerjee, S.M, 1935, The Buddhist Philosophy of Universal Flux-An Exposition of the Philosophy of Critical Realism as Expounded by the School of Dignaga, University of Calcutta, Calcuta; reimpreso por Motilaa Banarsidass, Delhi, 1975. 
Newton-Smith, W.H., 1981, The Rationality of Science, Routledge and Kegan Paul, Londres.

Oetke, C., 1996, "Ancient Indian Logic as a Theory of Non-Monotonic Reasoning", Journal of Indian Philosophy, vol. 24, pp. 447-539.

Piaget, J., 1972, Psychology and Epistemology-Towards a Theory of Knowledge, Penguin University Books, Penguin, Harmondsworth.

Pickering, A., 1995, The Mangle of Practice-Time, Agency and Science, The University of Chicago Press, Chicago.

- (comp.), 1992, Science as Practice and Cultures, The University of Chicago Press, Chicago.

— 1984, Constructing Quarks, The University of Chicago Press, Chicago.

Porter, T.M., 1986, The Rise of Statistical Thinking 1820-1900, Princeton University Press, Princeton.

Potter, K.H., 1965, Presuppositions of India's Philosophies, Prentice-Hall, Nueva Delhi.

Randle, H.N., 1930, Indian Logic in the Early Schools, Oxford University Press, Londres.

Sastri, H.P., 1906, "History of Nyayasastra form Japanese Sources", Journal of the Asiatic Society, reimpreso en D. Chattopadhyaya 1978, vol. 2, pp. 97-101.

_, 1905 , "An Examination of the Nyaya-Sutras", Journal of the Asiatic Society, reimpreso en D. Chattopadhyaya 1978, vol. 2, pp. 88-96.

Scarre, G., 1989, Logic and Reality in the Philosophy of John Stuart Mill, Kluwer Academic Publishers, Dordrecht.

Shapin, S. y S. Schaffer, 1985, Leviathan and the Air Pump-Hobbes, Bolye and the Experimental Life, Princeton University Press, Princeton.

Sharma, Ch., 1960, A Critical Survey of Indian Philosophy, Rider, Londres.

Smart, N., 1964, Doctrine and Argument in Indian Philosophy, Geroge Allen and Unwin, Londres.

Stanovich, K.E., 1999, Who is Rational-Studies of Individual Differences in Reasoning, Lawrence Erlbaum, Londres.

Stcherbatsky, FTh., 1930-1932, Buddhist Logic, Bibliotheca Buddhica XXVI, Leningrado, 2 vols.

Stein, E., 1996, Without Good Reason-The Rationality Debate in Philosophy and Cognitive Science, Clarendon, Oxford.

Vidyabhusana, M.S.Ch., 1913, The Nyaya Sutras of Gotama, Panini Office, Allahabad; 2a. ed.: Oriental Book Reprint, Nueva Delhi, 1975.

Recibido el 18 de julio de 2002; aceptado el 29 de octubre de 2002 